\title{
Asynchronous Gathering Algorithms for Autonomous Mobile Robots with Lights
}

\author{
Rikuo NAKAI ${ }^{1}$, Yuichi SUDO ${ }^{2}$, and Koichi $\mathrm{WADA}^{3}$ \\ 1 Graduate School of Science and Engineering, Hosei University, \\ 2 Faculty of Computer and Information Sciences, Hosei University, \\ 3 Faculty of Science and Engineering, Hosei University
}

\begin{abstract}
We consider a Gathering problem for $n$ autonomous mobile robots with persistent memory called light in an asynchronous scheduler (ASYNC). It is well known that Gathering is impossible when robots have no lights in basic common models, if the system is semi-synchronous (SSYNC) or even centralized (only one robot is active in each time). It is known that Gathering can be solved by robots with 10 colors of lights in ASYNC. This result is obtained by combining the following results. (1) The simulation of SSYNC robots with $k$ colors by ASYNC robots with $5 k$ colors [7], and (2) Gathering is solved by SSYNC robots with 2 colors [27].

In this paper, we improve the result by reducing the number of colors and show that Gathering can be solved by ASYNC robots with 3 colors of lights. We also show that we can construct a simulation algorithm of any unfair SSYNC algorithm using $k$ colors by ASYNC robots with $3 k$ colors, where unfairness does not guarantee that every robot is activated infinitely often. Combining this simulation and the Gathering algorithm by SSYNC robots with 2 colors [27], we obtain a Gathering algorithm by ASYNC robots with 6 colors. Our main result can be obtained by reducing the number of colors from 6 to 3 .
\end{abstract}

\section{Introduction}

\subsection{Background and Motivation}

The computational issues of autonomous mobile entities have been the object of much research in distributed computing. In this paper, we focus on mobile objects operating on a two-dimensional Euclidean space but there are several research on three-dimensional spaces and graphs [16]. Each robot operate in Look-Compute-Move ( $L C M)$ cycles. In the Look phase, an entity, viewed as a point and usually called robot, obtains a snapshot of the space; in the Compute phase it executes its algorithm (the same for all robots) using the snapshot as input; it then moves towards the computed destination in the Move phase. Repeating these cycles, the robots are able to collectively perform some tasks and solve some problems. The research interest has been on determining the impact that internal capabilities (e.g., memory, communication) and external conditions (e.g., synchrony, activation scheduler) have on the solvability of a problem. 
We also explore such weakest capabilities to solve the task. The problem considers in this paper is Gathering, which is one of the most fundamental tasks of autonomous mobile robots. Gathering is the process of $n$ mobile robots, initially located on arbitrary positions, meeting within finite time at a location, not known a priori. When there are only two robots, this task is called Rendezvous. Since Gathering and Rendezvous are simple but essential problems, they have been intensively studied, and a number of possibility and/or impossibility results have been shown under the different assumptions[1,2,4,6,9,10,14,19-25]. The solvability of Gathering and Rendezvous depends on the activation schedule and the synchronization level. Usually three basic types of schedulers are identified, the fully synchronous (FSYNC), the semi-synchronous (SSYNC) and the asynchronous (ASYNC) ${ }^{4}$. Gathering and Rendezvous are trivially solvable in FSYNC and the basic model. However, these problems cannot be solved in SSYNC without any additional assumptions [15], and the same is true in ASYNC. In particular, Gathering is not solvable even in a restricted subclass of SSYNC scheduler, where exactly one robot is activated in each round and always in the same order (called ROUND-ROBIN) [9]. If all robots are initially located on different positions (called distinct Gathering), this version of the problem is not solvable even in the CENT scheduler, in which exactly one robot is activated in each round $[9]$.

In [7], persistent memory called light has been introduced to reveal relationship between ASYNC and SSYNC and they show asynchronous robots with lights equipped with a constant number of colors, are strictly more powerful than semi-synchronous robots without lights: for any algorithm $\mathcal{A}$ designed for semisynchronous robots (without colors), they give a simulation algorithm by which asynchronous robots with 5 colors simulate an execution of $\mathcal{A}$. Rendezvous can be solved by robots with lights without any other additional assumptions [7, 17, 28]. Gathering is also solvable by robots with lights and it can be solved by robots with 2 colors of lights in SSYNC [27]. The power of lights to solve other problems are discussed in [11-13].

\subsection{Our Contribution}

In this paper, we study Gathering algorithms by robots with lights in the most realistic schedulers, ASYNC and some of the weakest conditions in term of computational power. As for Gathering algorithms in ASYNC, the following results are known; Cielieback et al. [6] solves the distinct Gathering for more than two robots with weak multiplicity detection, where the distinct gathering means all robots are initially placed in different positions, and weak multiplicity detection helps a robot to identify multiple occurrences of robots at a single point. Bhagat et al. [3] solves a gathering problem for five or more robots under the additional constraint to minimize the maximum distance traversed by any robots, with weak multiplicity detection or 4 colors of lights. Both algorithms work without

\footnotetext{
${ }^{4}$ In addition to these basic models, the new model semi-asynchronous (SAsync) [5] is recently proposed to reveal the gap between SSYNC and ASYNC.
} 
any extra assumptions like agreements of coordinate systems, unit distance and chirality and rigidity of movement, but they solve some constrained gathering problem. Gathering can be solved by ASYNC robots with 10 colors of lights by using the following two results;

(a) Any algorithm in SSYNC using $k$ colors of light can be simulated by ASYNC robots with $5 k$ colors of lights [7], and

(b) A Gathering algorithm is constructed by SSYNC robots with 2 colors of lights [27].

Since the algorithm shown in [27] needs the chirality assumption but no any other extra assumptions, the obtained algorithm works only with chirality.

This paper improves the result just stated above by reducing the number of colors and shows that Gathering can be solved by ASYNC robots with 3 colors of lights in no any other extra assumptions except chirality as follows;

1. We construct a simulation algorithm of any unfair SSYNC algorithm using $k$ colors by ASYNC robots with $3 k$ colors of lights, where unfair SSYNC is that the adversary makes enabled robots (changing its color or moving a different location) active in SSYNC. We have reduced the number of colors used in the simulation to $3 k$ from $5 k$, although the simulated algorithms are limited to ones working in unfair SSYNC. Since many robots algorithms seem to work in unfair SSYNC if it works in (fair) SSYNC, this simulation is interesting in itself and can be used to reduce the number of colors used in algorithms working in ASYNC.

2. We show that the Gathering algorithm with 2 colors of light shown in [27] can still work in unfair SSYNC. Hence we obtained that Gathering can be solved by ASYNC robots with 6 colors of lights in no any other extra assumptions except chirality. The Gathering algorithm of [27] is divided into two sub-algorithms. The first one makes a configuration that all robots are located on one straight line from any initial configuration and the second one is a Gathering algorithm from any initial configuration such that all robots are located on the straight line. The first one needs no lights but assumption of chirality and the second one need no extra assumptions and uses 2 colors. We show that the both algorithms can work in unfair SSYNC by defining a potential function for each algorithm and showing that each function becomes monotonically decreasing for any behaviour of each algorithm.

3. We improve the number of colors used in the algorithm into 3. Since the second algorithm uses 2 colors in SSYNC, the resultant algorithm have 6 colors. Thus in order to reduce the number of colors, we directly construct a 3-color Gathering algorithm from any configuration such that all robots are located on the straight line in ASYNC. Combining with the simulation of the first algorithm, we have obtained an ASYNC Gathering algorithm with 3 colors of light.

We have used the method by combining the simulation of SSYNC robots by ASYNC robots and algorithms working in SSYNC to reduce the number 
of colors used in the resultant algorithm. Proving the correctness of algorithms working in ASYNC is complicated and error-prone. However, our combination method can reduce the complexity of proving the correctness since the proof of working in ASYNC is divided into the proof of correctness of the simulation and the simulated algorithm working in SSYNC, both of which are easier than that in ASYNC.

\section{$2 \quad$ Model and Preliminaries}

\subsection{The Basics}

The systems considered in this paper consist of a team $R=\left\{r_{0}, \cdots, r_{n-1}\right\}$ of computational entities moving and operating in the Euclidean plane $\mathbb{R}^{2}$. Viewed as points, and called robots, the entities can move freely and continuously in the plane. Each robot has its own local coordinate system and it always perceives itself at its origin; there might not be consistency between these coordinate systems. A robot is equipped with sensorial devices that allows it to observe the positions of the other robots in its local coordinate system.

The robots are identical: they are indistinguishable by their appearance and they execute the same protocol. The robots are autonomous, without a central control.

At any point in time, a robot is either active or inactive. Upon becoming active, a robot $r_{i}$ executes a Look-Compute-Move (LCM) cycle performing the following three operations:

1. Look: The robot activates its sensors to obtain a snapshot of the positions occupied by robots with respect to its own coordinate system ${ }^{5}$. The snapshot of $r_{i}$ is denoted as $\mathcal{S} \mathcal{S}_{i}$.

2. Compute: The robot executes its algorithm using the snapshot as input. The result of the computation is a destination point.

3. Move: The robot moves in a straight line toward the computed destination but the robot may be stopped by an adversary before reaching the computed destination. In this case, the movement is called non-rigid. Otherwise, it is called rigid. When stopped before reaching its destination in the non-rigid movement, a robot moves at least a minimum distance $\delta>0$. If the distance to the destination is at most $\delta$, the robot can reach it. We assume non-rigid movement throughout the paper. If the destination is the current location, the robot stays still.

When inactive, a robot is idle. All robots are initially idle. The amount of time to complete a cycle is assumed to be finite, and the Look operation is assumed to be instantaneous.

There might not be consistency between the local coordinate systems and their unit of distance. The absence of any a-priori assumption on consistency of the local coordinate systems is called disorientation.

${ }^{5}$ This is called the full visibility (or unlimited visibility) setting; restricted forms of visibility have also been considered for these systems. 
The robots are said to have chirality if they share the same circular orientation of the plane (i.e., they agree on "clockwise" direction). If there is chirality, then there exists a unique circular ordering of locations occupied robots [26]. Thus, for each edge of the convex hull obtained by locations of $n$ robots $(n \geq 3)$, all robots can agree with the right vertex of the edge.

\subsection{The Models}

Different models, based on the same basic premises defined above, have been considered in the literature and we will use the following two models.

In the most common model, $\mathcal{O B L O} \mathcal{T}$, the robots are silent: they have no explicit means of communication; furthermore they are oblivious: at the start of a cycle, a robot has no memory of observations and computations performed in previous cycles.

In the other common model, $\mathcal{L U M \mathcal { I }}$, each robot $r_{i}$ is equipped with a persistent visible state variable $\ell_{i}$, called light, whose values are taken from a finite set $C$ of states called colors (including the color that represents the initial state when the light is off). The colors of the lights can be set in each cycle by $r_{i}$ at the end of its Compute operation. A light is persistent from one computational cycle to the next: the color is not automatically reset at the end of a cycle; the robot is otherwise oblivious, forgetting all other information from previous cycles. In $\mathcal{L U M \mathcal { M }}$, the Look operation produces a colored snapshot; i.e., it returns the set of pairs (position, color) of the other robots ${ }^{6}$. Note that if $|C|=1$, then the light is not used; thus, this case corresponds to the $\mathcal{O B L O} \mathcal{T}$ model.

We denote by $\ell_{i}(t)$ the color of light $r_{i}$ has at time $t$ and $p_{i}(t) \in \mathbb{R}^{2}$ the position occupied by robot $r_{i}$ at time $t$ represented in some global coordinate system. A configuration $\mathcal{C}(t)$ at time $t$ is a multi-set of $n$ pairs $\left(\ell_{i}(t), p_{i}(t)\right)$, each defining the color of light and the position of robot $r_{i}$ at time $t$. When no confusion arises, $\mathcal{C}(t)$ is simply denoted by $\mathcal{C}$.

If a configuration is that robots are located on a line segment connecting $p$ and $q$ (denoted as $p q$ ), this configuration is denoted by a regular-expression-like sequence of colors robots have from the endpoint $p$ to the other endpoint $q$. Formally we define color-configurations for a configuration of line segment $p q$ as follows; Let $\mathcal{C}(t)$ be the line-segment configuration at time $t$.Color-configurations for $\mathcal{C}(t)$ are defined as (0)-(3) as follows;

(0) Factor $f$ is defined as either $\alpha,(\alpha \mid \beta)$ or $(\alpha|\beta| \gamma)$, where $\alpha, \beta$ and $\gamma$ are colors and $(\alpha \mid \beta)$ and $(\alpha|\beta| \gamma))$ denote $\alpha$ or $\beta$ and $\alpha, \beta$ or $\gamma$, respectively. Color(s) which robots at a point have are denoted as $f$. Let $f, g$ and $h$ be factors.

(1) $f g$ denotes a configuration that all robots at $p$ have colors $f$, all robots at $q$ have colors $g$, and there are no robots inside the segment.

(2) $f g^{+} h$ denotes a configuration that all robots at $p$ have colors $f$, all robots at $q$ have colors $h$, and there exists at least one point inside the segment where all robots located there have colors $g$.

\footnotetext{
${ }^{6}$ If (strong) multiplicity detection is assumed, the snapshot is a multi-set.
} 
(3) $f g_{m} h$, if all robots at $p$ have colors $f$, all robots at $q$ have colors $h$, and all robots at the mid-point of the segment have colors $g$ and there are no robots except on the three locations.

If a color-configuration is one of $(1) \sim(3)$, it is denoted as $f g^{*} h$. Let $\operatorname{dis}(\mathcal{C}(t))$ denote the length of the segment in the configuration $\mathcal{C}(t)$. The color-configuration for $\mathcal{C}(t)$ is denoted as $c c(\mathcal{C}(t))$ and the number of points which have color $\alpha$ in $\mathcal{C}(t)$ is denoted as $\#_{\alpha}(\mathcal{C}(t))$. We also use this notation for a snapshot of robot.

In Section 4, color-configurations defined here are used, and in addition, one abuse of notation is used as follows. Letting $f, g$, and $h$ be factors, $f^{+} g h^{*}$ denotes that all robots at $p$ have colors $f$, all robots at $q$ have colors $g$ or $h$, and all robots inside the segment have colors $f, g$, and $h$.

\subsection{The Schedulers}

With respect to the activation schedule of the robots, and the duration of their LCM cycles, the fundamental distinction is between the asynchronous and synchronous settings.

In the synchronous setting (SSYNC), also called semi-synchronous, time is divided into discrete intervals, called rounds; in each round some robots are activated simultaneously, and perform their LCM cycle in perfect synchronization.

A popular synchronous setting which plays an important role is the fullysynchronous setting (FSYNC), where every robot is activated in every round; that is, the activation scheduler has no adversarial power.

In the asynchronous setting (ASYNC), there is no common notion of time, each robot is activated independently of the others, the duration of each phase is finite but unpredictable and might be different in different cycles. In this paper, we are concerned with ASYNC and we assume the following; In a Look operation, a snapshot of the environment is taken at some time $t_{L}$ and we say that the Look operation is performed at time $t_{L}$. Each Compute operation of $r_{i}$ is assumed to be done at time $t_{C}$ and the color of its light $\ell_{i}(t)$ and its pending destination $d e s_{i}$ are both set to the computed values for any time greater than $t_{C}{ }^{7}$. When the movement in a Move operation begins at time $t_{B}$ and ends at $t_{E}$, we say that it is performed during interval $\left[t_{B}, t_{E}\right]$, and the beginning (resp. ending) of the movement is denoted by Move ${ }_{B E G I N}$ (resp. Move END $_{\text {) occurring }}$ at time $t_{B}$ (resp. $t_{E}$ ). In the following, Compute, Move ${ }_{B E G I N}$ and Move END $_{\text {are }}$ abbreviated as Comp, $M_{B}$ and $M_{E}$, respectively. When a cycle has no actual movement (i.e., robots only change color and their destinations are the current positions), we can equivalently assume that the Move operation in this cycle is omitted, since we can consider the Move operation to be performed just before the next Look operation.

\footnotetext{
7 Note that if some robot performs a Look operation at time $t_{C}$, then it observes the former color and if it does at time $t_{C}+\epsilon(\forall \epsilon>0)$, then it observes the newly computed color.
} 
Without loss of generality, we assume the set of time instants at which the robots start executions of Look, Comp, $M_{B}$ and $M_{E}$ to be $\mathbb{N}$. We also assume the followings for each operation.

1. Comp operation is performed instantaneously at integer time $t_{C}$ and if some robot performs a Look operation at time $t_{C}$, then it observes the former color and if it does at time $t_{C}+1$, then it observes the newly computed color.

2. When the movement in a Move operation begins at $t_{B}$ and ends at $t_{E} \geq$ $t_{B}+1$ and if a robot performs a Look operation at time $t_{B}$ then it observes the location before moving and it does at time $t\left(t_{B}+1 \leq t \leq t_{E}\right)$, then it observes any location on the half-open line segment between one before moving (inclusive) and the destination (exclusive) satisfying the following condition, letting $p_{t}$ be the location of the moving robot at time $t$, for times $t$ and $t^{\prime}$ such that $t_{B}+1 \leq t<t^{\prime} \leq t_{E}$, it holds that $\operatorname{dis}\left(p_{t_{B}}, p_{t}\right)<\operatorname{dis}\left(p_{t_{B}}, p_{t^{\prime}}\right)$, where $\operatorname{dis}(p, q)$ denotes the distance between $p$ and $q$. The selected location is assumed to be determined by adversary. Also if it does at time $t_{E}+1$, it observes the destination.

In SSYNC and ASYNC settings, the selection of which robots are activated is made by an adversarial scheduler, whose only limit is that every robot must be activated infinitely often (i.e., it is a fair scheduler). We also consider an unfair scheduler. When a robot becomes active and performs the LCM cycle, the robot is enabled if it changes its color and/or the computed destination is different from the current position. The unfair scheduler does not guarantee that every robot is activated infinitely often. It is only guaranteed that if there is one or more enabled-robots at a time $t$, at least one enabled-robot will be activated or become non-enabled at some time $t^{\prime}>t$. Note that in a computation under this scheduler, an enabled-robot may not be activated until it becomes the only enabled robot.

\section{Simulating Algorithms in unfair SSYNC by ASYNC $\mathcal{L U M \mathcal { I }}$ robots}

In this section, we show that any $\mathcal{O B L O T}$ algorithm working in unfair SSYNC can be simulated by $\mathcal{L U M \mathcal { I }}$ robots with 3 colors in ASYNC.

\subsection{Simulation in ASYNC for Algorithms in unfair SSYNC}

We show an algorithm in ASYNC that simulates algorithms in unfair SSYNC. The algorithm is shown in Algorithm 1. The algorithm in square brackets indicates that it is given as input of a robot and simulated. Its transitions of colors of light is shown in Figure 1.The algorithm in square brackets indicates that it is given as input of a robot $r_{i}$ and simulated. Let $A_{\text {unfair }}$ be a simulated algorithm in unfair SSYNC. When a robot $r$ is enabled at a configuration $\mathcal{C}(t)$ in algorithm $A$, we say that $r$ is $A$-enabled at $\mathcal{C}(t)$. Our simulating algorithm uses light with 3 colors, $\mathrm{S}$ (tay), $\mathrm{M}$ (ove), and $\mathrm{E}$ (nd). We use the notation $\forall$ col 


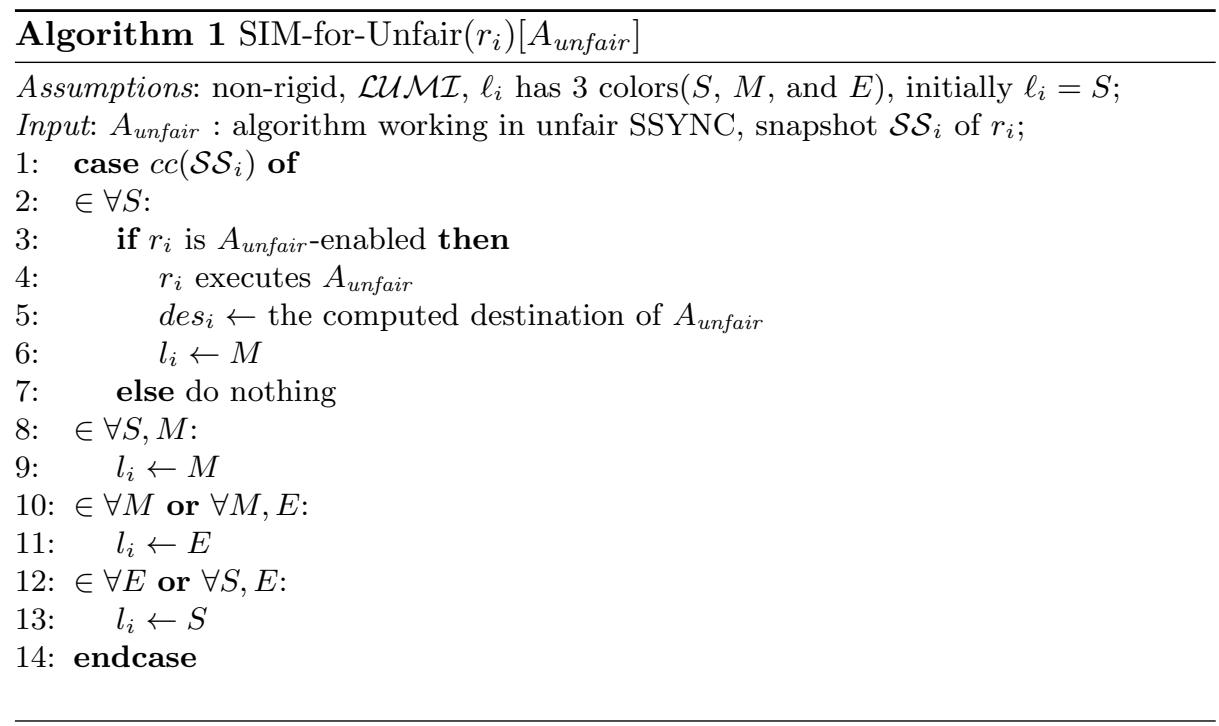

for a color col denoting a set of configuration such that all robots have color $\mathrm{col}$. We also use the notation $\forall \mathrm{col}_{1}, \mathrm{col}_{2}$ for colors $\mathrm{col}_{1}$ and $\mathrm{col}_{2}$ denoting a set of configurations such that each robot has color $\mathrm{col}_{1}$ or $\mathrm{col}_{2}$ and there exists at least one robot with color $\mathrm{col}_{1}$ and there exists at least one robot with color $\mathrm{col}_{2}$. Initial configuration is in $\forall S$. This algorithm repeats a color-cycle, that is, the transition of $\forall S \rightarrow \forall M \rightarrow \forall E$. When the configuration is in $\forall S$, since $A_{\text {unfair- }}$ enabled-robots exist, some $A_{\text {unfair-enabled-robots that become active among }}$ those execute $A_{\text {unfair }}$, change their colors to $M$, and move to the computed destination. While they move after changing their colors to $M$, other robots change their colors to $M$ until the configuration becomes one in $\forall M$. Note that when the configuration is in $\forall M$, some robots may be still moving. After the robots reach a configuration in $\forall M$, the robots change their colors to $E$ until the configuration becomes one in $\forall E$. In the same way, the configuration changes from a configuration in $\forall E$ to a configuration in $\forall S$. This cycle is repeated until the robots reach a configuration where no robot is $A_{\text {unfair-enabled and all robots }}$ are colored $S$. Each time a configuration in $\forall S$ where one or more robots are $A_{\text {unfair-enabled is reached, at least one of them becomes active and performs }}$ $A_{\text {unfair }}$ observing the same configuration. In consequence, this algorithm can simulate algorithms in unfair SSYNC.

We show that SIM-for-Unfair $\left(r_{i}\right)$ simulates $A_{\text {unfair }}$ correctly in ASYNC.

Lemma 1. Let the configuration be in $\forall S$ at time $t_{S}$ and let $R_{e}$ be a set of

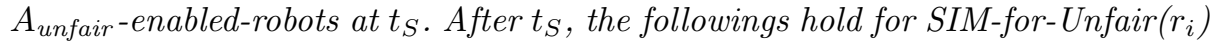
if $R_{e} \neq \emptyset$.

(1) There is a time $t_{M}>t_{S}$ at which the configuration is in $\forall M$. 


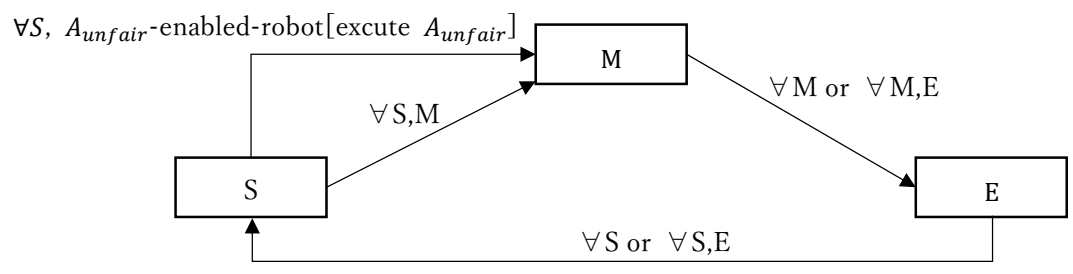

Fig. 1. Transition Graph for SIM-for-Unfair $\left(r_{i}\right)$.

(2) There are a time $t_{f}\left(t_{S}<t_{f}<t_{M}\right)$ and a non-empty subset $R_{e}^{\prime}$ of $R_{e}$ such that all robots in $R_{e}^{\prime}$ perform $A_{\text {unfair }}$ observing the same configuration and all robots in $R_{e}-R_{e}^{\prime}$ do nothing between $t_{S}$ and $t_{f}$.

Proof. Let $t_{f}$ be a time at which the first robot changes its color from $S$ to $M$ after $t_{S}$. Setting $R_{e}^{\prime}$ be a set of $A_{\text {unfair }}$-enabled-robots activated between $t_{S}$ and $t_{f}$, since $\mathcal{C}\left(t_{S}\right)$ is not changed between $t_{S}$ and $t_{f}$, each $A_{\text {unfair-enabled- }}$ robot in $R_{e}^{\prime}$ observes the same configuration $\mathcal{C}\left(t_{S}\right)$, the robot performs lines 4-6 in Algorithm 1. They perform $A_{\text {unfair }}$ with the same configuration and change their colors to $M$. Any other robot than $R_{e}^{\prime}$ does nothing even if it is activated between $t_{S}$ and $t_{f}$. Then (2) holds.

Activated robots observing some robot with $M$ after $t_{f}+1$ change their colors to $M$ (lines 8-9). This continues until $\forall M$ is satisfied and there exists a time $t_{M}$ stated in (1) due to the fairness of ASYNC. Note that all robots do not finish their $L C M$-cycle at time $t_{M}$.

Lemma 2. If the configuration is in $\forall M$ at time $t_{M}$, there is a time $t_{E}$ at which it is in $\forall E$.

Proof. If the configuration is in $\forall M$ at time $t_{M}$, activated robots after $t_{M}$ change their colors from $M$ to $E$ until the configuration in $\forall E$. Since all robots become active after $t_{M}$ by the fairness of ASYNC, there is a time $t_{E}$ at which the configuration is in $\forall E$.

Note that since robots with $M$ stay when changing their colors from $M$ to $E$, the configuration at $t_{E}$ is unchanged until some robots are activated after $t_{E}$.

The following lemma holds similarly.

Lemma 3. If the configuration is in $\forall E$ at time $t_{E}$, there is a time $t_{S}$ at which it is in to $\forall S$.

Using Lemmas 1-3, we can verify that algorithm SIM-for-Unfair $\left(r_{i}\right) \operatorname{simulates}$ $\mathcal{O B} \mathcal{L O T}$-algorithm $A_{\text {unfair }}$ in unfair SSYNC correctly in ASYNC with 3 colors of $\mathcal{L U M \mathcal { I }}$-light, and if $A_{\text {unfair }}$ uses $k$ colors of $\mathcal{L U} \mathcal{M I}$-light, SIM-for-Unfair $\left(r_{i}\right)$ uses $3 k$ colors. Then the following theorem is obtained.

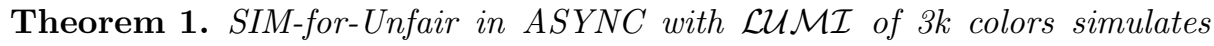

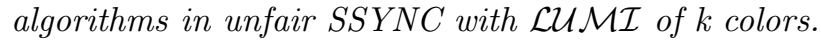




\subsection{Gathering Algorithm with Simulation}

In order to show that an algorithm $A$ works in unfair SSYNC, we use a concept of potential function for $A$, which represents how close current configuration is to the final configuration. A potential function $f_{A}$ for algorithm $A$ is a function from time $t(\in \mathbb{N})$ to feature value obtained from configuration $\mathcal{C}(t)$ for the algorithm $A$, which is taken from a total ordered set. If the potential function $f_{A}$ for algorithm $A$ working in SSYNC is monotonically decreasing, that is, $f_{A}(t)>f_{A}(t+1)$ for any $t$, we can show that the algorithm $A$ can work in unfair SSYNC.

In the subsequent subsections, we show that the Gathering algorithm with two colors of light shown in SSYNC in [27] can still work in unfair SSYNC by constructing potential functions.

The Gathering algorithm [27] is divided into two sub-algorithms. The first one (called ElectOneLDS) obtains a configuration that all robots are located on one straight line segment (called onLDS) from any initial configuration, and the second one (called $\mathcal{L U M \mathcal { I }}$-Gather) is a Gathering algorithm from any initial configuration of onLDS.

We obtain an algorithm with SIM-for-Unfair, ElectOneLDS, and $\mathcal{L U M \mathcal { I }}$ Gather by replacing the line 4 in Algorithm 1 with the line

if not onLDS then ElectOneLDS $\left(r_{i}\right)$ else $\mathcal{L U M \mathcal { M }}$-Gather $\left(r_{i}\right)$.

This algorithm simulates ElectOneLDS $\left(r_{i}\right)$ until onLDS is attained and once onLDS is obtained it simulates $\mathcal{L U M \mathcal { I }}$-Gather and Gathering is completed. If

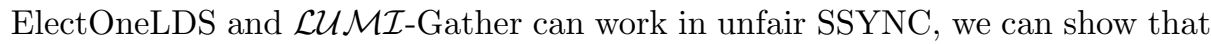
the combined algorithm solves Gathering in ASYNC. In the subsequent subsections, We will show that ElectOneLDS and $\mathcal{L U M \mathcal { M }}$-Gather can still work in unfair SSYNC by constructing monotonically decreasing potential functions for the both algorithms.

\subsection{ElectOneLDS works in unfair SSYNC}

ElectOneLDS [27] is the algorithm producing onLDS from any initial configuration and is shown in algorithm $2^{8}$. The algorithm changes the control of robots by the configuration that is symmetric or asymmetric and contractible or not. Let $\mathcal{C H}(\mathcal{C}(t))$ be the convex hull obtained by a configuration $\mathcal{C}(t)$ at time $t$. $\mathcal{C}(t)$ is symmetric if all edges of the convex hull $\mathcal{C H}(\mathcal{C}(t))$ have the same length, otherwise it is asymmetric. $\mathcal{C}(t)$ is contractible if $\mathcal{C}(t)$ satisfies the following conditions;

(i) $\mathcal{C}(t)$ is symmetric, and all robots are on the vertices or on the center of $\mathcal{C H}(\mathcal{C}(t))$, or

(ii) $\mathcal{C}(t)$ is asymmetric, and all robots are on the vertices or edges.

\footnotetext{
8 The original algorithm in [27] is slightly different, where in the case of consecutive minimum edges (line 9) all the edges are contracted but here only the rightmost edge is contracted. This change has no effect for the correctness but is necessary to work in ASYNC.
} 


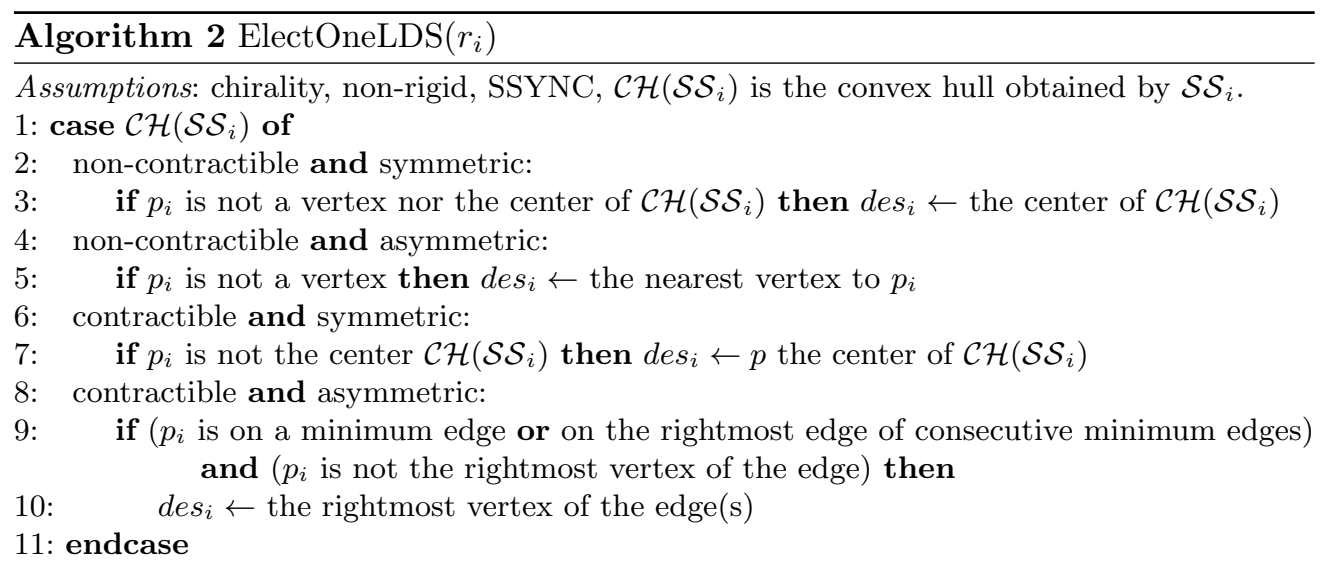

Figure 2 shows the transitions between configurations. We define a potential function $f: \mathbb{N} \rightarrow(\mathbb{R})^{2} \times \mathbb{N} \times(\mathbb{R})^{2}$ as follows; The range of $f$ is a 5-dimensional vector and $f$ is denoted by $<f_{1}^{\text {area }}, f_{2}^{\text {Cdist }}, f_{3}^{\# \text { in }}, f_{4}^{\text {Edist }}, f_{5}^{\text {Vdist }}>$. The order of values of the range set is taken with the lexicographic order.

Let $\mathcal{C H}(\mathcal{C}(t))$ be the convex hull obtained from $\mathcal{C}(t)$ and its vertices are denoted as $\left\langle v_{0}, \ldots, v_{k-1}\right\rangle$, where the vertices are located counter-clockwise and the edge $v_{0} v_{1}$ is the longest and $v_{0}$ is the rightmost and topmost vertex in the global coordinate system. Let $p_{i}(1 \leq i \leq n)$ denote the location of $r_{i}$.

1. The function value $f_{1}^{a r e a}(t)$ is the area of $\mathcal{C H}(\mathcal{C}(t))$.

2. If $\mathcal{C}(t)$ is symmetric and not onLDS, the function value $f_{2}^{C d i s t}(t)$ is the sum of distances between the center of $\mathcal{C H}\left(\mathcal{C}(t)\right.$ ) (denoted as $p_{c}$ ) and all robots' locations. Otherwise it is 0 . That is,

$$
f_{2}^{C d i s t}(t)= \begin{cases}\sum_{i=0}^{n-1} \operatorname{dis}\left(p_{c}, p_{i}\right) & (\mathcal{C}(t) \text { is symmetric }) \\ 0 & (\mathcal{C}(t) \text { is asymmetric }) .\end{cases}
$$

3. If $\mathcal{C}(t)$ is asymmetric, the function value $f_{3}^{\# i n}(t)$ is the number of robots inside $\mathcal{C H}(\mathcal{C}(t))$. Otherwise, it is 0 .

4. If $\mathcal{C}(t)$ is asymmetric, the function value $f_{4}^{E d i s t}(t)$ the sum of distances traversing the vertices of $\mathcal{C H}(\mathcal{C}(t))$ counter-clockwise from the rightmost and topmost vertex $v_{0}$ to the locations of robots on $\mathcal{C H}(\mathcal{C}(t))$.

Let $\left\langle v_{0}^{\prime}, v_{1}^{\prime}, \ldots, v_{k^{\prime}-1}^{\prime}>\right.$ be a sequence of the locations of robots on $\mathcal{C H}(\mathcal{C}(t))$ such that the locations are listed counter-clockwise from the rightmost and topmost vertex $v_{0}$. Note that $v_{0}^{\prime}=v_{0}$.

$$
f_{4}^{\text {Edist }}(t)= \begin{cases}0 & (\mathcal{C}(t) \text { is symmetric }) \\ \sum_{i=0}^{k^{\prime}-1} \operatorname{dist}\left(v_{0}, v_{i}^{\prime}\right) & (\mathcal{C}(t) \text { is asymmetric }) .\end{cases}
$$

5. If $\mathcal{C}(t)$ is asymmetric, the function value $f_{5}^{V d i s t}(t)$ is the sum of distances from all robots' location to the nearest vertex of $\mathcal{C H}(\mathcal{C}(t))$. That is, letting 
$n v_{i}$ be the nearest vertex of $\mathcal{C H}(\mathcal{C}(t))$ from the location $p_{i}$ of robot $r_{i}$.

$$
f_{5}^{V d i s t}(t)= \begin{cases}0 & (\mathcal{C}(t) \text { is symmetric }) \\ \sum_{i=0}^{n-1} d i s\left(n v_{i}, p_{i}\right) & (\mathcal{C}(t) \text { is asymmetric })\end{cases}
$$

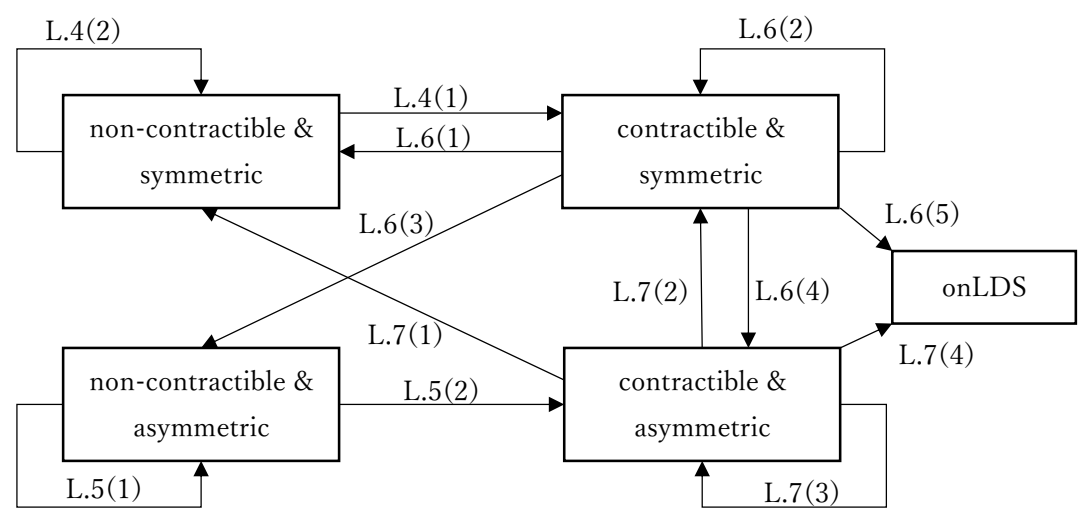

Fig. 2. Transition Graph for ElectOneLDS

Lemma 4. If $\mathcal{C}(t)$ is symmetric and non-contractible, it holds that $f(t)>f(t+$ 1) and

(1) $\mathcal{C}(t+1)$ is symmetric and contractible, or

(2) $\mathcal{C}(t+1)$ is symmetric and non-contractible.

Proof. At time $t$, robots at points except vertices and the center of $\mathcal{C H}(\mathcal{C}(t))$ become enabled. If all enabled-robots move to the center, the configuration becomes symmetric and contractible. Thus, although $f_{1}^{a r e a}(t)$ is unchanged, and $f_{2}^{C d i s t}(t)$ decreases. Otherwise, the configuration remains symmetric and noncontractible. Then (2) is also holds.

Lemma 5. If $\mathcal{C}(t)$ is asymmetric and non-contractible, it holds that $f(t)>f(t+$ 1) and

(1) $\mathcal{C}(t+1)$ is asymmetric and non-contractible, or

(2) $\mathcal{C}(t+1)$ is asymmetric and contractible.

Proof. At time $t$, a robot $r_{i}$ that locates at a point except vertices and edges of $\mathcal{C H}(\mathcal{C}(t))$ becomes enabled and moves to the nearest vertex $n v_{i}$.

(1) Unless all robots inside $\mathcal{C H}(\mathcal{C}(t))$ reach $n v_{i}$ at $t+1, f_{1}^{\text {area }}(t), f_{3}^{\# \text { in }}(t)$ and $f_{4}^{\text {Edist }}(t)$ are unchanged because $\mathcal{C H}(\mathcal{C}(t))$ and the number of points on $\mathcal{C H}(\mathcal{C}(t))$ 
Table 1. In ElectOneLDS, change(dec. or inc.) of functions' values from $f(t)$ to $f(t+1)$.

\begin{tabular}{|c|c|c|c|}
\hline $\mathcal{C}(t)$ & enabled-robots on ( $\rightarrow$ destination) & $\mathcal{C}(t+1)$ & $\begin{array}{c}\text { change of function } \\
\text { (dec.,inc.) }\end{array}$ \\
\hline s\&nc & points except vertices $(\rightarrow$ the center of $\mathcal{C H}(\mathcal{C}(t)))$ & s\&nc or s\&c & $\left(f_{2}\right.$, none $)$ \\
\hline a\&nc & points inside $\mathcal{C H}(\mathcal{C}(t))(\rightarrow$ the nearest vertex $)$ & a\&nc or a\&c & $\begin{array}{c}\left(f_{3} \& f_{5}, f_{4}\right) \\
\left(f_{5}, \text { none }\right)\end{array}$ \\
\hline s\&c & vertices $(\rightarrow$ the center of $\mathcal{C H}(\mathcal{C}(t)))$ & $\begin{array}{l}\text { s\&nc, s\&c, } \\
\text { a\&nc, a\&c } \\
\text { or onLDS }\end{array}$ & $\begin{array}{c}\left(f_{1}, \text { none }\right) \\
\left(f_{2}, \text { none }\right) \\
\left(f_{1} \& f_{2}, f_{3-5}\right) \\
\left(f_{1} \& f_{2}, \text { none }\right)\end{array}$ \\
\hline $\mathrm{a} \& \mathrm{c}$ & $\begin{array}{l}\text { non-consecutive minimum edge or } \\
\text { rightmost consecutive minimum edge } \\
(\rightarrow \text { the right endpoint of the edge })\end{array}$ & $\begin{array}{l}\text { s\&nc, s\&c, } \\
\text { a\&c, } \\
\text { or onLDS }\end{array}$ & $\begin{array}{c}\left(f_{1} \& f_{3-5}, f_{2}\right) \\
\left(f_{1} \& f_{4}, \text { none }\right) \\
\left(f_{4}, \text { none }\right) \\
\left(f_{1} \& f_{3-5}, \text { none }\right)\end{array}$ \\
\hline
\end{tabular}

Abbreviated notations: "s":symmetric, "as":aymmetric, "c":contractible, "nc":non-contractible, $f_{i}$ omits the superscript.

do not change. In this case, since there is at least one enabled-robot that moves to $n v_{i}, f_{5}^{V \text { dist }}(t)$ decreases, and $f(t)>f(t+1)$. Otherwise, since the number of points on $\mathcal{C H}(\mathcal{C}(t))$ increases, $f_{4}^{E d i s t}$ increases. However, the number of points inside $\mathcal{C H}(\mathcal{C}(t))$ decreases, and so $f_{3}^{\# i n}(t)$ and $f_{5}^{V \text { dist }}(t)$ decrease. Therefore, comparing in lexicographic order, we have $f(t)>f(t+1)$.

(2)In this case, all robots inside $\mathcal{C H}(\mathcal{C}(t))$ at $t$ reach to $n v_{i}$. Since the number of points on $\mathcal{C H}(\mathcal{C}(t))$ increases, $f_{4}^{E d i s t}(t)$ increases. However, the number of points inside $\mathcal{C H}(\mathcal{C}(t))$ decreases, and $f_{3}^{\# i n}(t)$ and $f_{5}^{V d i s t}(t)$ decrease. Thus, we have $f(t)>f(t+1)$.

Lemma 6. If $\mathcal{C}(t)$ is symmetric and contractible, it holds that $f(t)>f(t+1)$ and

(1) $\mathcal{C}(t+1)$ is symmetric and non-contractible,

(2) $\mathcal{C}(t+1)$ is symmetric and contractible,

(3) $\mathcal{C}(t+1)$ is asymmetric and non-contractible,

(4) $\mathcal{C}(t+1)$ is asymmetric and contractible, or

(5) $\mathcal{C}(t+1)$ is onLDS.

Proof. At time $t$, robots that locate at vertices of $\mathcal{C H}(\mathcal{C}(t))$ become enabled and move to the center $p_{c}$ of $\mathcal{C H}(\mathcal{C}(t))$.

(1)If $\mathcal{C H}(\mathcal{C}(t))$ does not change, there is one or more enabled-robot that move to $p_{c}$, and $f_{2}^{C d i s t}(t)$ decreases. Otherwise, there are robots at a vertex of $\mathcal{C H}(\mathcal{C}(t))$ at $t$ that reach at $p_{c}$. Then $\mathcal{C H}(\mathcal{C}(t))$ shrinks, and $f_{1}^{\text {area }}(t)$ decreases.

(2)If $\mathcal{C H}(\mathcal{C}(t))$ does not change, there is a robot that reaches $p_{c}$, and $f_{2}^{C d i s t}(t)$ decreases. Otherwise, robots at each vertex moves by the same distance, or there are robots at a vertex of $\mathcal{C H}(\mathcal{C}(t))$ that reach at $p_{c}$. Then $\mathcal{C H}(\mathcal{C}(t))$ shrinks, and $f_{1}^{\text {area }}(t)$ decreases. 
(3) There are robots at a vertex of $\mathcal{C H}(\mathcal{C}(t))$ that move inside $\mathcal{C H}(\mathcal{C}(t))$. Then $\mathcal{C H}(\mathcal{C}(t))$ shrinks, and $f_{1}^{\text {area }}(t)$ decreases.

(4) There are robots at a vertex of $\mathcal{C H}(\mathcal{C}(t))$ that move inside $\mathcal{C H}(\mathcal{C}(t))$. Then $\mathcal{C H}$ shrinks, and $f_{1}^{\text {area }}(t)$ decreases.

(5)When all robots not on a vertex at $t$ arrived at the center $p_{c}$ of $\mathcal{C H}(\mathcal{C}(t))$, or $\mathcal{C H}(\mathcal{C}(t))$ has the regular polygon that has a diagonal through its center, and all robots not on a diagonal at $t+1$ arrived at $p_{c}$ of $\mathcal{C H}(\mathcal{C}(t))$, onLDS is created at $t+1$. Since onLDS is symmetric, and $f_{1}^{\text {area }}(t+1)$ and $f_{2}^{C d i s t}(t+1)$ is 0 , $f(t+1)=<0,0,0,0,0>$.

Lemma 7. If $\mathcal{C}(t)$ is asymmetric and contractible, it holds that $f(t)>f(t+1)$ and

(1) $\mathcal{C}(t+1)$ is symmetric and non-contractible,

(2) $\mathcal{C}(t+1)$ is symmetric and contractible,

(3) $\mathcal{C}(t+1)$ is asymmetric and contractible,

(4) $\mathcal{C}(t+1)$ is onLDS.

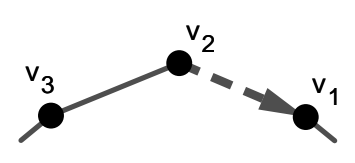

(a)

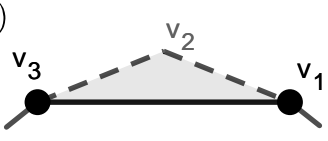

(b)

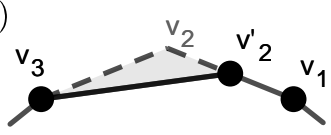

(c)

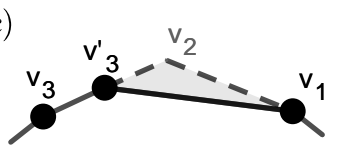

$(d)$

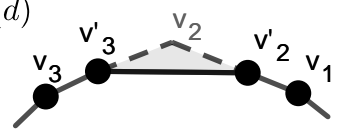

Fig. 3. In cases of shrunken $\mathcal{C H}(\mathcal{C}(t+1))$ that is asymmetric and contractible

Proof. At time $t$, robots on the rightmost edge of consecutive minimum edges or a non-consecutive minimum edge of $\mathcal{C H}(\mathcal{C}(t))$ except the right endpoint become enabled and move to the right endpoint. If $\mathcal{C}(t+1)$ is symmetric, since enabledrobots move along the minimum edge, the edge remains the minimum edge until the edge is contracted. Symmetric configuration is obtained by contracting the edges that is the rightmost edge of consecutive minimum edges or a nonconsecutive minimum edge. Then, since $\mathcal{C H}(\mathcal{C}(t+1))$ is obtained by removing some vertices from $\mathcal{C H}(\mathcal{C}(t)), \mathcal{C H}(\mathcal{C}(t))$ shrinks, and $f_{1}^{\text {area }}(t)$ decreases. This proves (1) and (2).

If $\mathcal{C}(t+1)$ is asymmetric, enabled-robots move along the minimum edge. Figure 3 shows the cases where the contracted edge is $v_{1} v_{2}$, and $\mathcal{C H}(\mathcal{C}(t+1))$ and $\mathcal{C H}(\mathcal{C}(t))$ are different due to all robots on $v_{2}$ move toward $v_{1}$. In (a), there are not robots on the edge $v_{2} v_{3}$, and robots on $v_{2}$ reach $v_{1}$. $\operatorname{In}(\mathrm{b})$, there are not robots on the edge $v_{2} v_{3}$, and robots on $v_{2}$ move toward $v_{1}$ and stopped 
on the edge. In (c), there is a robot on the edge $v_{2} v_{3}$, and robots on $v_{2}$ reach $v_{1}$. In $(\mathrm{d})$, there is a robot on the edge $v_{2} v_{3}$, and robots on $v_{2}$ move toward $v_{1}$ and stopped on the edge. In these cases, $\mathcal{C H}(\mathcal{C}(t))$ shrinks, and $f_{1}^{\text {area }}(t)$ and $f_{4}^{E d i s t}(t)$ decrease.Even if $\mathcal{C H}(\mathcal{C}(t))$ does not change, at least one enabled-robot moves along the right vertex, and $f_{4}^{E d i s t}(t)$ decreases. Therefore, $(3)$ holds.

When $\mathcal{C}(t+1)$ is onLDS, $\mathcal{C}(t)$ is either the triangle that has one or two minimum edges or a rectangle except square. If $\mathcal{C}(t)$ is the triangle that has one or two minimum edges, and there are no robots on the left-hand edge of contracted edge at $t+1$, onLDS is made by contracting one edge. Note that in the case of the triangle that has two minimum edges, only the rightmost edge of the two is contracted. Since onLDS is symmetric, and $f_{1}^{\text {area }}(t)$ and $f_{2}^{C d i s t}(t)$ are 0 , we have $f(t+1)=<0,0,0,0,0>$. If $\mathcal{C}(t)$ is a rectangle except square, onLDS is made by contracting the two minimum edge. Since onLDS is symmetric, and $f_{1}^{\text {area }}(t+1)$ and $f_{2}^{C d i s t}(t+1)$ are $0, f(t+1)=<0,0,0,0,0>$. Therefore, (4) holds.

Table 1 summarizes Lemmas 4-7. Elements of the potential function on $\mathbb{R}$ decrease depending on $\delta$ even if the area of $\mathcal{C H}$, because robots under non-rigid movement can move at least a distance $\delta$. Therefore, the next theorem holds.

Theorem 2. The potential function $f$ for ElectOneLDS is monotonically decreasing.

\section{$3.4 \mathcal{L U} \mathcal{M I}$-Gather works in unfair SSYNC}

$\mathcal{L U M \mathcal { I }}$-Gather [27] is the Gathering algorithm where it starts with any onLDS configuration and it uses $2 \mathcal{L U} \mathcal{M I}$-colors $(A$ and $B$ ) and works in SSYNC. $\mathcal{L U} \mathcal{M I}$-Gather is shown in Algorithm 3 and the transition between colorconfigurations is depicted in Figure 4. The outline of Algorithm 3 is as follows;

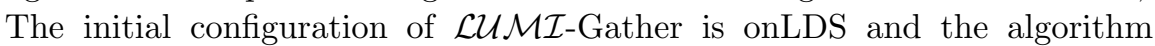
makes $A A$ via $A A^{+} A$ and robots at endpoints move to the midpoint after changing its colors to $B$. Then the color-configuration becomes $A B_{m} A$ or $A B^{+} A$, $A B^{*} B$ or $B B^{*} A$, or $B B^{*} B$ or $B B_{m} B$.

(1) For the case that the number of point with $A$ is one $\left(A B^{*} B\right.$ or $\left.B B^{*} A\right)$, the points with $A$ is the Gathering point and so robots with $A$ stay and robots with $B$ move to the point with $A$ and Gathering is attained.

(2) For the case that the number of points with $A$ is zero $\left(B B^{*} B\right)$, robots at endpoints change their colors to $A$, the color configuration is $A B^{*} A(A A$, $A B_{m} A$ or $\left.A B^{+} A\right)$. The first case is the same as the initial color configuration, but the distance between endpoints is decreased by at least $2 \delta$, and the second case is treated in (3).

(3) For the case that the number of points with $A$ is two $\left(A B_{m} A\right.$ or $\left.A B^{+} A\right)$, after making $A B_{m} A$ from $A B^{+} A$, robots at endpoints move to the midpoint after changing their colors to $B$. Then Gathering is attained, or the color 
configuration becomes one same as the first transition from the initial configuration but the distance between endpoints is decreased by at least $2 \delta$. If the distance between endpoints is at most $2 \delta$, since robots at endpoints can reach the midpoint this repetition is finished in finite times and Gathering is attained.

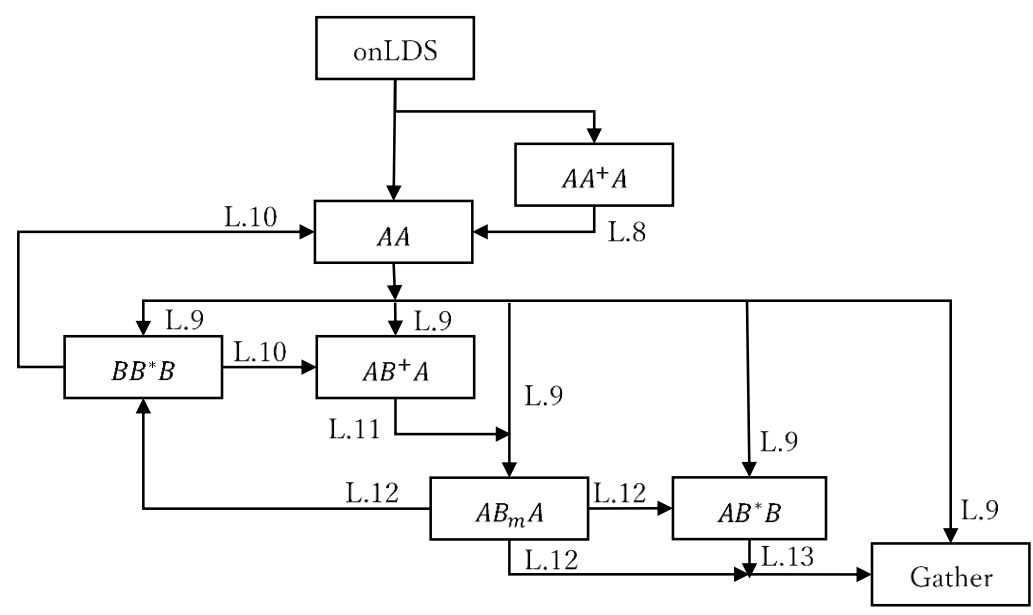

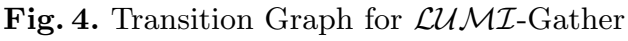

We define a potential function $g: \mathbb{N} \rightarrow(\mathbb{R} \cup\{\infty\})^{3} \times \mathbb{N} \times \mathbb{R}$ as follows; The range of $g$ is a 5-dimensional vector and $g$ is denoted by $<g_{1}^{\text {Adist }}, g_{2}^{\text {Edist }}, g_{3}^{\text {Mdist }}, g_{4}^{\# B}, g_{5}^{\text {NEdist }}>$.

1. If $\#_{A}(\mathcal{C}(t))=1$ then the function vaule $g_{1}^{\text {Adist }}(t)$ is the sum of distances between the position with color $A$ (denoted as $p_{A}$ ) and all robot's position, otherwise, it is $\infty$. That is,

$$
g_{1}^{\text {Adist }}(t)= \begin{cases}\sum_{i=0}^{n-1} \operatorname{dis}\left(p_{A}, p_{i}\right) & \left(\#_{A}(\mathcal{C}(t))=1\right) \\ \infty & \left(\#_{A}(\mathcal{C}(t))>1\right)\end{cases}
$$

2. In the case that $\#_{A}(\mathcal{C}(t))=0$ or 2 , the function value $g_{2}^{E d i s t}(t)$ is the distance between two endpoints. If $\#_{A}(\mathcal{C}(t))=1$, then it is 0 , otherwise it is $\infty$.

$$
g_{2}^{\text {Edist }}(t)= \begin{cases}0 & \left(\#_{A}(\mathcal{C}(t))=1\right) \\ \operatorname{dis}(\mathcal{C}(t)) & \left(\#_{A}(\mathcal{C}(t))=0,2\right) \\ \infty & \left(\#_{A}(\mathcal{C}(t)) \geq 3\right)\end{cases}
$$

3. In the case that $\#_{A}(\mathcal{C}(t))=0$ or 2 , the function value $g_{3}^{M d i s t}(t)$ is the sum of distances between every robot's position and the midpoint of LDS (denoted as $\left.p_{m}\right)$. If $\#_{A}(\mathcal{C}(t))=1$, then it is 0 , otherwise it is $\infty$. That is,

$$
g_{3}^{M d i s t}(t)= \begin{cases}0 & \left(\#_{A}(\mathcal{C}(t))=1\right) \\ \sum_{i=0}^{n-1} \operatorname{dis}\left(p_{m}, p_{i}\right) & \left(\#_{A}(\mathcal{C}(t))=0,2\right) \\ \infty & \left(\#_{A}(\mathcal{C}(t)) \geq 3\right)\end{cases}
$$




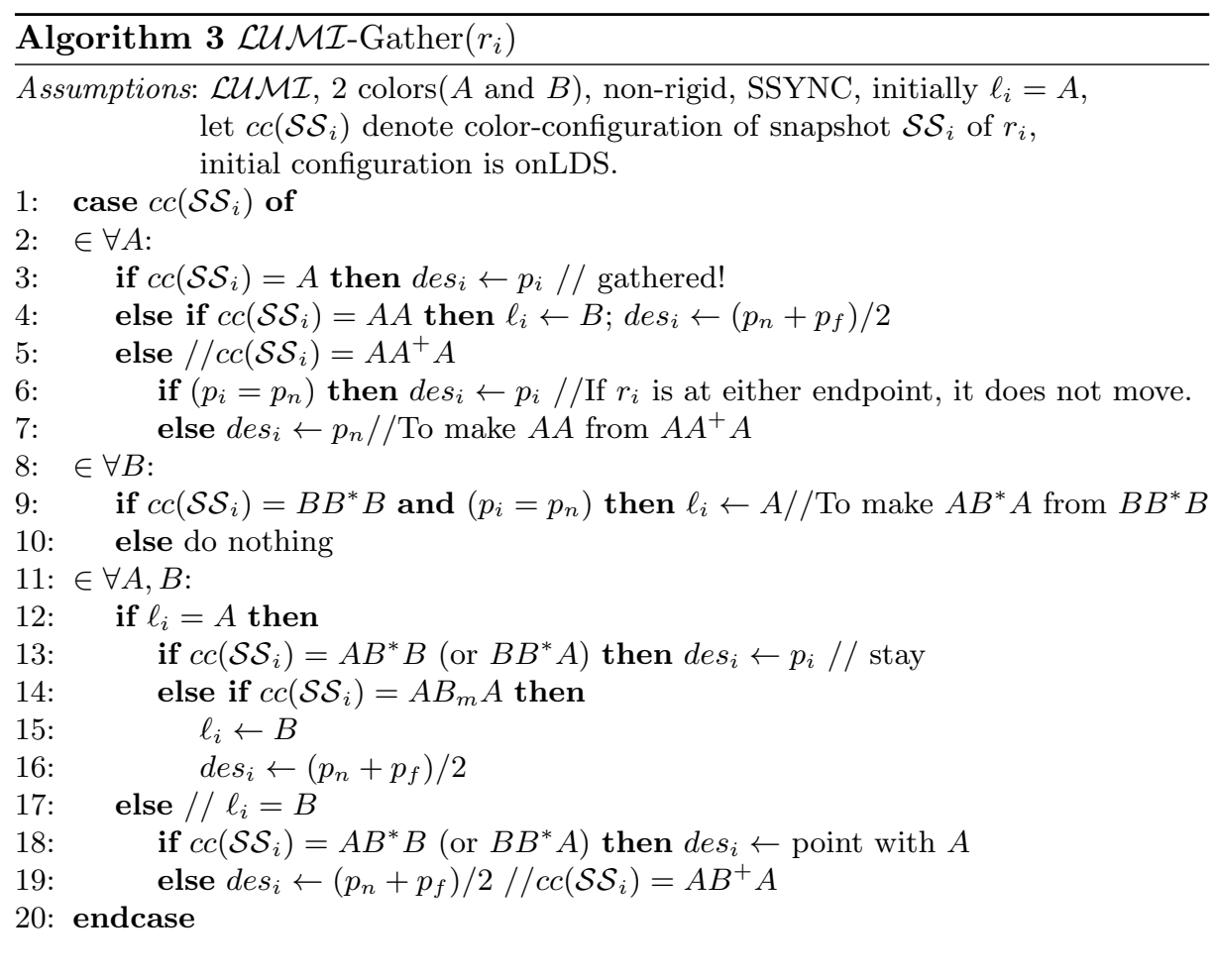

4. In the case that $\#_{A}(\mathcal{C}(t))=0$ or 2 , the function value $g_{4}^{\# B}(t)$ is the number of robots with color $B$. If $\#_{A}(\mathcal{C}(t))=1$, then it is 0 , otherwise it is $\infty$. Let $R_{B}=\{r \mid r$ has color $B\}$.

$$
g_{4}^{\# B}(t)= \begin{cases}0 & \left(\#_{A}(\mathcal{C}(t))=1\right) \\ \left|R_{B}\right| & \left(\#_{A}(\mathcal{C}(t))=0,2\right) \\ \infty & \left(\#_{A}(\mathcal{C}(t)) \geq 3\right)\end{cases}
$$

5. If $\#_{A}(\mathcal{C}(t)) \leq 3$, the function value $g_{5}^{N E d i s t}(t)$ is the sum of distances between every robot's position $p_{i}$ and the nearest endpoint to $p_{i}$ (denoted as $n p_{i}$ ), otherwise it is 0 .

$$
g_{5}^{N E d i s t}(t)= \begin{cases}0 & \left(\#_{A}(\mathcal{C}(t))>3\right) \\ \sum_{i=0}^{n-1} \operatorname{dis}\left(n p_{i}, p_{i}\right) & \left(\#_{A}(\mathcal{C}(t)) \leq 3\right)\end{cases}
$$

Note that following the definition of $g$, if $\left|\mathcal{P}_{A}(\mathcal{C}(t))\right|=1$, then

$$
g(\mathcal{C}(t))=<\sum_{i=1}^{n}\left|p_{A}-p_{i}\right|, 0,0,0,0>\mathcal{C}(t),
$$


if $\left|\mathcal{P}_{A}(\mathcal{C}(t))\right|=0$, or $\left|\mathcal{P}_{A}(\mathcal{C}(t))\right|=2$, then

$$
g(\mathcal{C}(t))=<\infty,\left|p_{r}-p_{l}\right|, \sum_{i=1}^{n}\left|p_{i}-p_{m}\right|,\left|R_{B}\right|, 0>\mathcal{C}(t)
$$

, if $\left|\mathcal{P}_{A}(\mathcal{C}(t))\right| \geq 3$, then

$$
g(\mathcal{C}(t))=<\infty, \infty, \infty, \infty, \sum_{i=1}^{n}\left|p_{n}-p_{i}\right|>\mathcal{C}(t)
$$

Table 2. In $\mathcal{L U M \mathcal { I }}$-Gather, change(dec. or inc.) of functions' values from $g(t)$ to

\begin{tabular}{|c|c|c|c|}
\hline$c c(\mathcal{C}(t))$ & enabled-robots on $(\rightarrow$ destination\&color $)$ & $c c(\mathcal{C}(t+1))$ & $\begin{array}{c}\text { change of function } \\
\text { (dec.,inc.) }\end{array}$ \\
\hline$A A^{+} A$ & $\begin{array}{c}\text { points except endpoints } \\
\left(\rightarrow \text { the nearest } p_{e} \& \text { unchanged }\right)\end{array}$ & $\begin{array}{l}A A^{+} A \\
\text { or } A A\end{array}$ & $\begin{array}{c}\left(g_{5}, \text { none }\right) \\
\left(g_{2-5}, \text { none }\right)\end{array}$ \\
\hline$A A$ & endpoints $(\rightarrow$ the midpoint $\& B)$ & $\begin{array}{c}A B^{+} A, A B_{m} A, \\
A B^{*} B, \\
B B^{*} B, \\
\text { or Gather }\end{array}$ & $\begin{array}{c}\left(g_{3}, g_{4}\right) \\
\left(g_{1-4}, \text { none }\right) \\
\left(g_{2}, g_{4}\right) \\
\left(g_{2} \& g_{3}, g_{4}\right)\end{array}$ \\
\hline$B B^{*} B$ & endpoints $(\rightarrow$ stay $\& A)$ & $\begin{array}{c}A B^{*} A \\
\text { or } A B^{*} B\end{array}$ & $\begin{array}{c}\left.\text { ( } g_{4}, \text { none }\right) \\
\left(g_{1-4}, \text { none }\right)\end{array}$ \\
\hline$A B^{+} A$ & $\begin{array}{c}\text { points except endpoints } \\
(\rightarrow \text { the midpoint\&unchanged })\end{array}$ & $\begin{aligned} & A B^{+} A \\
& \text { or } A B_{m} B \\
&\end{aligned}$ & $\left(g_{3}\right.$, none $)$ \\
\hline$A B_{m} A$ & endpoints $(\rightarrow$ the midpoint $\& B)$ & $\begin{array}{c}A B^{+} A, \\
A B^{+} B \\
B B^{*} B \\
\text { or Gather }\end{array}$ & $\begin{array}{c}\left(g_{3}, g_{4}\right) \\
\left(g_{1-4}, \text { none }\right) \\
\left(g_{2}, g_{4}\right) \\
\left(g_{2} \& g_{3}, g_{4}\right)\end{array}$ \\
\hline$A B^{*} B$ & $\begin{array}{c}\text { points except endpoint with } A \\
(\rightarrow \text { the endpoint with } A \& \text { unchanged })\end{array}$ & $\begin{array}{c}A B^{*} B \\
\text { or Gather }\end{array}$ & $\left(g_{1}\right.$, none $)$ \\
\hline
\end{tabular}
$g(t+1)$.

Function $g_{i}$ omits the superscript.

Lemma 8. If $c c(\mathcal{C}(t))=A A^{+} A$, it holds that $g(t)>g(t+1)$.

Proof. At time $t$, robots locating inside onLDS become enabled and move to the nearest endpoint $p_{n}$. In the case of $\#_{A}(\mathcal{C}(t)) \geq 3$, there is at least one enabledrobot that moves to $p_{n}$, and $g_{5}^{N E d i s t}(t)$ decreases. In the case of $\#_{A}(\mathcal{C}(t))=2$, $g_{2}^{E d i s t}(t)$ decreases from $\infty$ to the distance of the segment. Therefore, this lemma holds.

Lemma 9. If $c c(\mathcal{C}(t))=A A$, it holds that $g(t)>g(t+1)$. 
Proof. At time $t$, robots at the endpoints become enabled and move to the midpoint $p_{m}$ of the two endpoints. In the case that $\#_{A}(\mathcal{C}(t+1))=2$, the endpoints do not change, and at least one enabled robot changes its color to $B$ and moves to $p_{m}$. Thus, $g_{4}^{\# B}(t)$ increases, but $g_{3}^{M d i s t}(t)$ decreases. In the case that $\#_{A}(\mathcal{C}(t+1))=1, g_{1}^{\text {Adist }}(t)$ decreases from $\infty$ to $\sum_{i=0}^{n-1} d i s\left(p_{a}, p_{i}\right)$. In the case that $\#_{A}(\mathcal{C}(t+1))=0$ and not Gather, all robots change their colors to $B$ and move to $p_{m}$. Then $g_{4}^{\# B}(t)$ increase, and $g_{3}^{M d i s t}(t)$ may also increase, but $g_{2}^{E d i s t}(t)$ decreases. In the case that $\#_{A}(\mathcal{C}(t+1))=0$ and Gather, since $g_{2}^{\text {Edist }}(t+1), g_{3}^{\text {Mdist }}(t+1)$, and $g_{4}^{\# B}(t+1)$ are $0, g(t+1)=<\infty, 0,0,0,0>$. Therefore, $g(t)>g(t+1)$.

Lemma 10. If $c c(\mathcal{C}(t))=B B^{*} B$, it holds that $g(t)>g(t+1)$.

Proof. At time $t$, robots at the endpoints become enabled and change their colors to $A$. If $\#_{A}(\mathcal{C}(t+1))=2$, clearly the number of robots with $A$ increases, and only $g_{4}^{\# B}(t)$ decreases. Otherwise $\#_{A}(\mathcal{C}(t+1))=1, g_{1}^{\text {Adist }}(t)$ decreases from $\infty$ to $\sum_{i=0}^{n-1} \operatorname{dis}\left(p_{a}, p_{i}\right)$. Therefore, $g(t)>g(t)$.

Lemma 11. If $c c(\mathcal{C}(t))=A B^{+} A$, it holds that that $g(t)>g(t+1)$.

Proof. Robots with $B$ at points except $p_{m}$ and the endpoints become enabled and move to $p_{m}$. Since robots at the endpoints are unchanged, it is enough to consider the case where $\#_{A}(\mathcal{C}(t))=2$. Since there is at least one enabled-robot that moves to $p_{m}$, only $g_{3}^{M d i s t}(t)$ decreases.

Lemma 12. If $c c(\mathcal{C}(t))=A B_{m} A$, it holds that that $g(t)>g(t+1)$.

Proof. Robots with $A$ at the endpoints become enabled, change their colors to $B$, and move to $p_{m}$. In the case of $\#_{A}(\mathcal{C}(t))=2$, robots at the endpoints are unchanged, and there is at least one enabled-robot that changes its color $B$ and moves. Since $g_{4}^{\# B}(t)$ increases, but $g_{3}^{M d i s t}(t)$ decreases.

In the case of $\#_{A}(\mathcal{C}(t))=1, g_{1}^{\text {Adist }}(t)$ decreases from $\infty$ to $\sum_{i=0}^{n-1} \operatorname{dis}\left(p_{a}, p_{i}\right)$. In the case of $\#_{A}(\mathcal{C}(t))=0$ and not Gather, all robots at the endpoints change their colors $B$ and move to $p_{m}$. Then although $g_{4}^{\# B}(t)$ increases and $g_{3}^{M d i s t}(t)$ may also increases, $g_{2}^{E d i s t}(t)$ decreases. In the case that $\#_{A}(\mathcal{C}(t+1))=0$ and Gather, since $g_{2}^{E \text { dist }}(t+1), g_{3}^{M \text { dist }}(t+1)$, and $g_{4}^{\# B}(t+1)$ are $0, g(t+1)=<$ $\infty, 0,0,0,0>$. Therefore, $g(t)>g(t+1)$.

Lemma 13. If $c c(\mathcal{C}(t))=A B^{*} B$, it holds that that $g(t)>g(t+1)$.

Proof. At time $t$, robots with color $B$ become enabled and move to the point with color $A$ (denoted as $p_{A}$ ). Then it is enough to consider the case where $\#_{A}(\mathcal{C}(t))=$ 1. Since there is at least one enabled-robot that moves to $p_{A}, g_{1}^{\text {Adist }}(t)$ decreases.

Table 2 summarizes Lemmas 8-13, which follow the next theorem.

Theorem 3. The potential function $g$ for $\mathcal{L U M \mathcal { M } - G a t h e r ~ i s ~ m o n o t o n i c a l l y ~ d e - ~}$ creasing. 
We obtain the following theorem by Theorems 1-3

Theorem 4. Gathering can be solved in $A S Y N C$ by $\mathcal{L U M \mathcal { I }}$ robots having 6 colors under non-rigid movement, and agreement of chirality.

In the next section, we can reduce the number of colors to three by construct a Gathering algorithm starting from OnLDS working in ASYNC with three colors.

\section{Gathering Algorithm in ASYNC with 3 colors}

In this section, we give a Gathering algorithm called 3-color-Gather-in-ASYNC working in ASYNC with 3 colors. The pseudocode is shown in Algorithm 4. This algorithm consists of two algorithms, where one is to make onLDS and uses the simulation of ElectOneLDS (SIM-for-Unfair[ElectOneLDS]), and the other is a Gathering algorithm from onLDS and does not use the simulation and is newly developed (called $\mathcal{L U} \mathcal{M I}$-Gather-in-ASYNC). As we will show in Corollary 1, in SIM-for-Unfair[ElectOneLDS], once a configuration becomes onLDS, it remains onLDS forever. Therefore, the algorithm works in ASYNC with 3 colors and therefore 3-color-Gather-in-ASYNC attains Gathering in ASYNC with 3 colors.

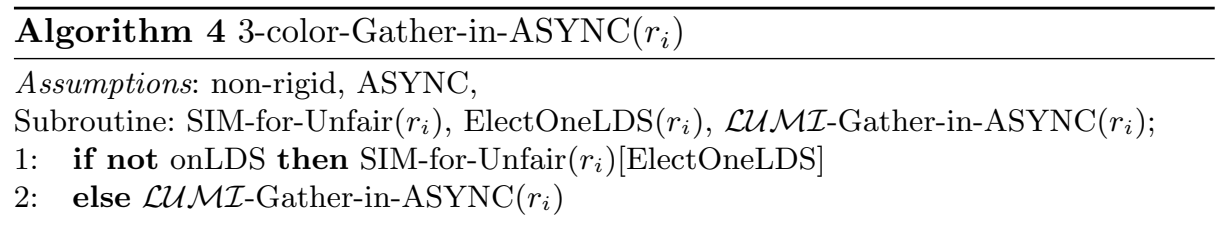

\subsection{Configurations becoming onLDS}

In 3-color-Gather-in-ASYNC, it is switched to $\mathcal{L U M \mathcal { I }}$-Gather-in-ASYNC from the simulation when the configuration becomes onLDS. We consider configurations which become onLDS when ElectOneLDS is simulated by SIM-for-Unfair.

Since we are concerned with $\mathrm{ASYNC}, \mathcal{C}(t)$ contains moving robots ${ }^{9}$ and/or robots having performed Look but not performing Compute. The former robots are said to be in pending move at $\mathcal{C}(t)$ and the latter robots are said to be in pending color at $\mathcal{C}(t)$ [8]. Then the following notations are introduced in colorconfigurations. In factor $f$ of a color-configuration for $\mathcal{C}(t)$, if some robots have the possibility to be in pending move or pending color at a position represented by $f$, the factor is denoted by $f[p m]$ and $f[p c \rightarrow \alpha]$, respectively. If there is possibility of robots being in pending move and in pending color, the factor is denoted by $f[p m, p c]$, where $p c \rightarrow \alpha$ shows that the color is changed to $\alpha$ when performing Compute. When robots in pending move with color $\alpha$ move to the destination $d$ in the factor $\alpha[\mathrm{pm}]$, we say that $\alpha[\mathrm{pm}]$ has destination $d$.

\footnotetext{
${ }^{9}$ Robots having performed Compute and not finishing Move yet.
} 
Lemma 14. If the configuration becomes onLDS at $t$ when SIM-for-Unfair simulates ElectOneLDS. It holds that

(1) $c c(\mathcal{C}(t))=S S^{*} S$,

(2) $c c(\mathcal{C}(t))=(S|S[p c \rightarrow M]| M \mid M[p m])(S|S[p c \rightarrow M]| M \mid M[p m])^{*}(S \mid S[p c \rightarrow$ $M]|M| M[p m])$ with at least one $M$,

(3) $c c(\mathcal{C}(t))=(M|M[p m, p c \rightarrow E]| E)(M|M[p m, p c \rightarrow E]| E)^{*}(M \mid M[p m, p c \rightarrow$ $E] \mid E)$ with at least one $M$,

(4) $c c(\mathcal{C}(t))=(S|S[p c \rightarrow M]| M)$ with at least one $M$, or

(5) $c c(\mathcal{C}(t))=(M|M[p c \rightarrow E]| E)$ with at least one $M$.

In (2) and (3), all $M[\mathrm{pm}]$ has a destination of a point on the straight line through onLDS in $\mathcal{C}(t)$.

Proof. If the initial configuration is already onLDS, trivially (1) holds. We first prove about $c c(\mathcal{C}(t))$. If $\mathcal{C}\left(t^{\prime}\right)$ is not onLDS for a time $t^{\prime}<t$, at least one enabledrobot has to reach its destination to become onLDS. Since it has color $M$, at least one $M$ exists in $\mathcal{C}(t)$. If there is a robot that has never performed $\mathrm{Com}$ pute until $\mathrm{t}, \mathcal{C}(t)$ is in the course of transition from configuration in $\forall S$ to configuration in $\forall M$. It is possible that there is a robot with $S$ looks a configuration in $\forall S$ before $t$, or a robot with $M$ is moving toward its destination of a point on onLDS. Hence, $c c(\mathcal{C}(t))$ is $(S|S[p c \rightarrow M]| M \mid M[p m])(S \mid S[p c \rightarrow$ $M]|M| M[p m]) *(S|S[p c \rightarrow M]| M[p m])$ with at least one $M$ or $(S|S[p c \rightarrow M]| M)$ with at least one $M$. Otherwise, the configuration becomes a configuration in $\forall M$ at say, $t_{\forall M}$ before $t$. Then robots except $A_{\text {unfair-enabled-robots changed their }}$ colors to $M$ at $t_{M}\left(t_{M}<t_{\forall M}<t\right)$, where $\mathcal{C}\left(t_{M}\right)$ has color $M$. If a robot with $M$ performs $L o o k$ between $t_{\forall M}$ to $t$, it tries to change its color to $E$. Since $\mathcal{C}(t)$ is in the course of transition from configuration in $\forall M$ to configuration in $\forall E$, and then $c c(\mathcal{C}(t))=(M|M[p m, p c \rightarrow E]| E)(M|M[p m, p c \rightarrow E]| E)^{*}(M \mid M[p m, p c \rightarrow$ $E] \mid E)$ with at least one $M$ or $(M|M[p c \rightarrow E]| E)$ with at least one $M$.

Next, we show that destinations of robots in pending move with $M$ are in onLDS. Let $t$ be a time at which a configuration is onLDS for the first time, and let the time $t_{B}=\max \left\{t_{S} \mid \mathcal{C}\left(t_{S}\right) \in \forall S, t_{S}<t\right\}$. At $t_{B}$, configurations are only the following cases.

(a) $\mathcal{C}\left(t_{B}\right)$ is asymmetric and contractible, and is a triangle that has one or two minimum edge,

(b) $\mathcal{C}\left(t_{B}\right)$ is asymmetric and contractible, and is a rectangle, or

(c) $\mathcal{C}\left(t_{B}\right)$ is symmetric and contractible.

For (a), if $\mathcal{C H}\left(\mathcal{C}\left(t_{B}\right)\right)$ has exactly one minimum edge, robots on the edge except the rightmost vertex become enabled at $t_{B}$. When all of them arrived at the rightmost vertex, onLDS is made. Hence, robots with $M$ must be stopped at $t$. If $\mathcal{C H}\left(\mathcal{C}\left(t_{B}\right)\right)$ has the two minimum edges, robots on a right-hand edge of the two and not on the rightmost vertex become enabled at $t_{B}$. When they arrived at the rightmost vertex, onLDS is made. Hence, robots with $M$ must be also stopped. 
For (b), robots on the minimum edges become enabled at $t_{B}$. When they all arrived at the rightmost vertex, onLDS is made. Hence, robots with $M$ must be stopped.

For (c), there are two cases where LDS is obtained at $t$. One is a line segment whose endpoints on a diagonal through the center of $\mathcal{C H}\left(\mathcal{C}\left(t_{B}\right)\right)$ (denoted as $\left.p_{c}\right)$, and the other is one connecting $p_{c}$ and a point on a diagonal through $p_{c}$. Let $x$ and $y$ be the diagonal for the former case and let $z$ be the endpoint except $p_{c}$ for the latter case. In the former case all robots on vertices other than $x$ and $y$ have reached at $p_{c}$ and are stopped at $t$. Robots at $x$ or $y$ are in pending color or pending move and their destination is $p_{c}$. Thus, their destinations are in onLDS. The latter case can be shown similarly.

Corollary 1. In SIM-for-Unfair[ElectOneLDS], if the configuration becomes onLDS from non-onLDS at time $t$, destination of any moving robot at $t$ is a point on the straight line through onLDS in $\mathcal{C}(t)$.

We will show that $\mathcal{L U M \mathcal { M }}$-Gather-in-ASYNC can work from the configurations shown in Lemma 14.

\subsection{Correctness of $\mathcal{L U M \mathcal { M }}$-Gather-in-ASYNC}

$\mathcal{L U M I}$-Gather-in-ASYNC(Algorithm 5) is an extension of Algorithm 3 so that it can work in ASYNC, and uses color-cycles similar to that of Algorithm 1. This algorithm use 3 colors $S, M$, and $E$ and its color-cycle repeats $\forall S(S S) \rightarrow \forall M \rightarrow$ $\forall E(E E) \rightarrow \forall S(S S)$. Notatins in parentheses indicate that the configuration is limited to two points. 


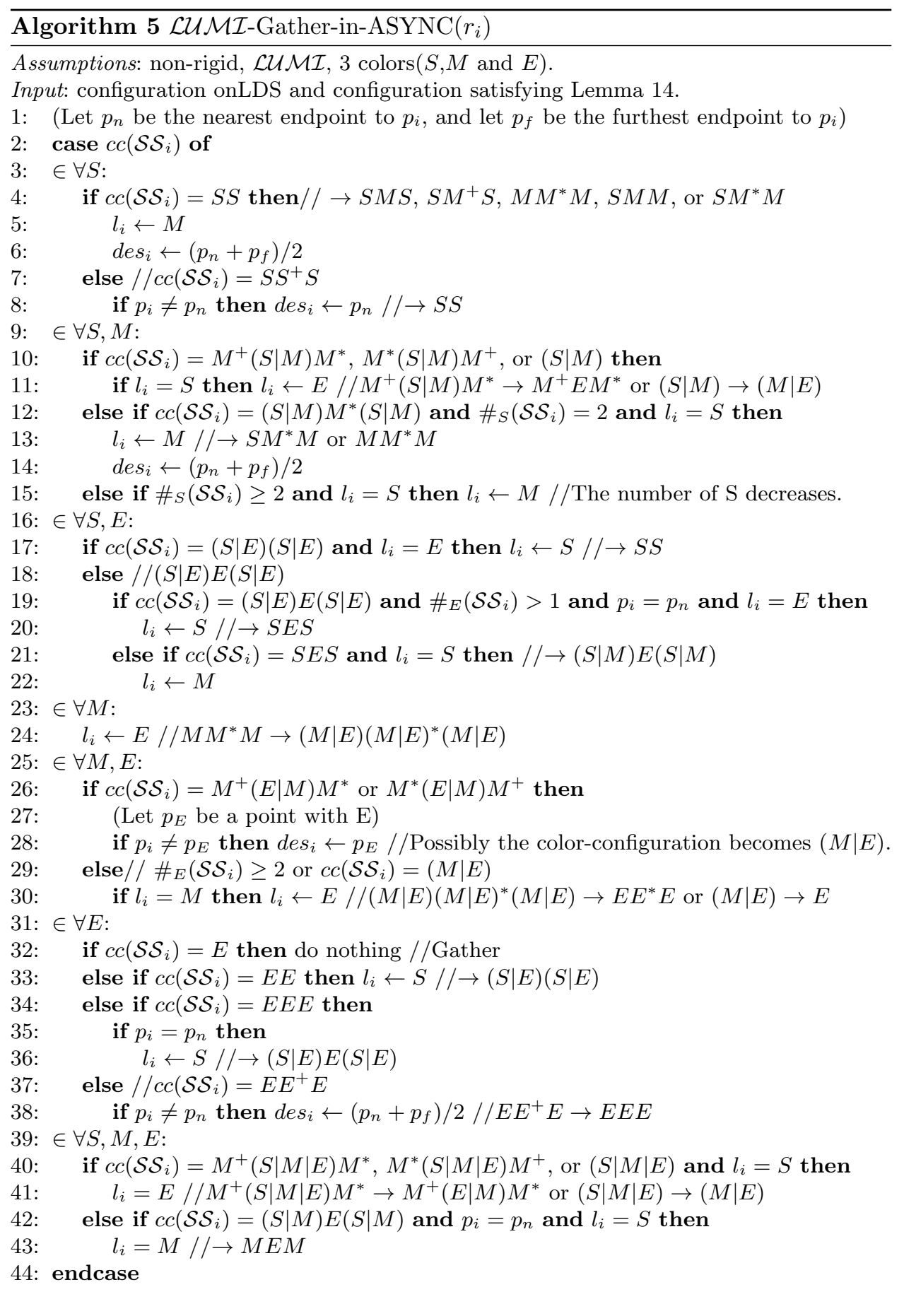




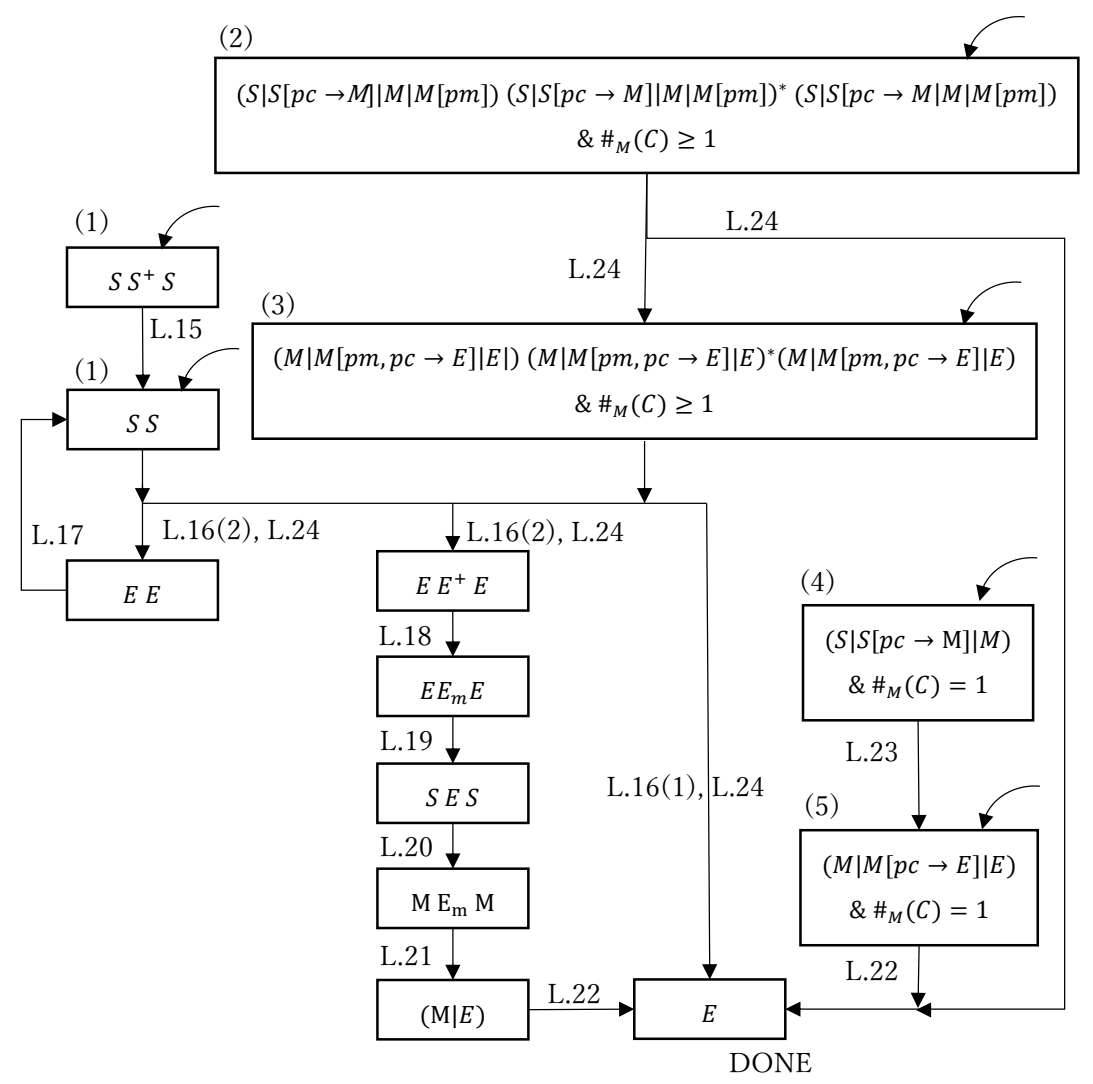

Fig. 5. Transition Graph for $\mathcal{L U M \mathcal { I }}$-Gather-in-ASYNC from $\mathcal{C}$ in Lemma 14.

In the algorithm, robots gather at the midpoint of some onLDS, or Gathering point, where configuration $\mathcal{C}$ has a Gathering point $p_{G}$ if and only if $\mathcal{C}$ is in $\forall M, E$, $\#_{E}(\mathcal{C})=1$ and $p_{G}$ has $E$. Thus, the aim of this algorithm to create Gathering point during color-cycles.

In $\forall S(S S) \rightarrow \forall M$, robots on the two points with $S$ change their colors to $M$ and move to the midpoint. Note that robots at the endpoints move to the midpoint only if the endpoints have $S$ and the color of the robots is $S$. Gathering point is created during transitions in color-cycles for the following cases;

(1) During $\forall S(S S) \rightarrow \forall M$, robots with $S$ look configuration $\mathcal{C}$ such that $\#_{S}(\mathcal{C})=$ 1.

(2) configuration $\mathcal{C}$ during $\forall M \rightarrow \forall E$.

(3) After configuration becomes $\forall E$ configuration $\mathcal{C}$ such that $\#_{E}(\mathcal{C}) \geq 3$.

We show how to create a Gathering point from each of (1),(2), and (3). 
For (1), a robot $r_{i}$ with $S$ changes its color to $E$ if $r_{i}$ looks the configuration with $\#_{S}(\mathcal{C})=1$. From the configuration it will make a configuration such that $\#_{E}(\mathcal{C})=1$ and $\forall M, E$, and then a Gathering point is created.

For (2), Gathering point is lost if there are more than one point with $E$. On the other hands, Gathering point is confirmed if there is only one point with $E$. Let $t_{M}$ be a time at which the configuration becomes $\forall M$, let $t_{E}\left(>t_{M}\right)$ be the first time at which some robot changes its color to $E$, and let $p_{E}$ be the location having robots with $E$. If there are not activated robots at points except $p_{E}$ between $t_{M}+1$ and $t_{E}$, robots activated after $t_{E}$ observe a configuration with $\#_{E}(\mathcal{C})=1$ and $\forall M, E$. Therefore, there are no robots that change their colors to $E$, and the Gathering point $p_{E}$ is confirmed.

In (3), robots with $E$ do not move, and the both endpoints are fixed and robots at points except the endpoints move to the midpoint. Thus the color-configuration becomes $E E E$. The transition of the configuration becomes $E E E \rightarrow S E S \rightarrow$ $M E M$, where the last configuration satisfies $\#_{E}(\mathcal{C})=1$ and in $\forall M, E$. This case also determines a Gathering point.

If the configuration becomes (1), (2) or (3), Gathering point is made. If the configuration does not become (1), (2) and (3), it becomes $E E$, will change $S S$ and again begins the next color-cycle $\forall S(S S) \rightarrow \forall M \rightarrow \forall E(E E) \rightarrow \forall S(S S)$. If color-cycles are repeated, the distance of the endpoints is reduced by at least $2 \delta$ in one cycle. Therefore the distance will become less than $2 \delta$ when the color configuration becomes $\forall S(S S)$. Then, if the configuration is in $\forall M$, robots with $M$ reach the midpoint, and Gathering is achieved. Transitions between color configurations in Algorithm 5 are shown in Figure 5. In this figure, boxes with numbers and $\curvearrowleft$ are starting configurations and the number corresponds to that in Lemma 14. Arrow labelled with L.n $(i)$ means it is proved in Lemma $n(i)$. "DONE" means Gathering is attained.

The following lemmas show transitions between color-configurations in Algorithm 5 .

Lemma 15. If $c c(\mathcal{C}(t))=S S^{+} S$, there is a time $t^{\prime}>t$ such that $c c\left(\mathcal{C}\left(t^{\prime}\right)\right)=S S$.

Proof. If robots on points except the endpoints of onLDS become active, they move to the nearest endpoint (line 8 ). While $\#_{S}(\mathcal{C}) \geq 3$, robots on the endpoints do nothing (line 9) until the color-configuration becomes $c c(\mathcal{C})=S S$. Therefore, there is a time t' at which $c c\left(\mathcal{C}\left(t^{\prime}\right)\right)=S S$.

Lemma 16. If $c c(\mathcal{C}(t))=S S$, there is a time $t^{\prime}>t$ such that

(1) $c c\left(\mathcal{C}\left(t^{\prime}\right)\right)=E$, or

(2) $c c\left(\mathcal{C}\left(t^{\prime}\right)\right)=E E^{*} E$ and $\operatorname{dis}(\mathcal{C}(t)) \leq \operatorname{dis}\left(\mathcal{C}\left(t^{\prime}\right)\right)-2 \delta$.

Proof. Transitions from $c c(\mathcal{C}(t))=S S$ are depicted in Fig. 6 .

Case(a) Transition from $c c(\mathcal{C}(t))=S S$.

We show that if $c c(\mathcal{C}(t))=S S$, there is a time $t_{\forall M}(>t)$ such that $c c\left(\mathcal{C}\left(t_{\forall M}\right)\right)=$ $(M \mid M[p m])(M \mid M[p m])^{*}(M \mid M[p m])$ and the destination of robots in $M[p m]$ is the midpoint of onLDS in $\mathcal{C}(t)$ (Case (c)) or a time $t_{S}(>t)$ such that 


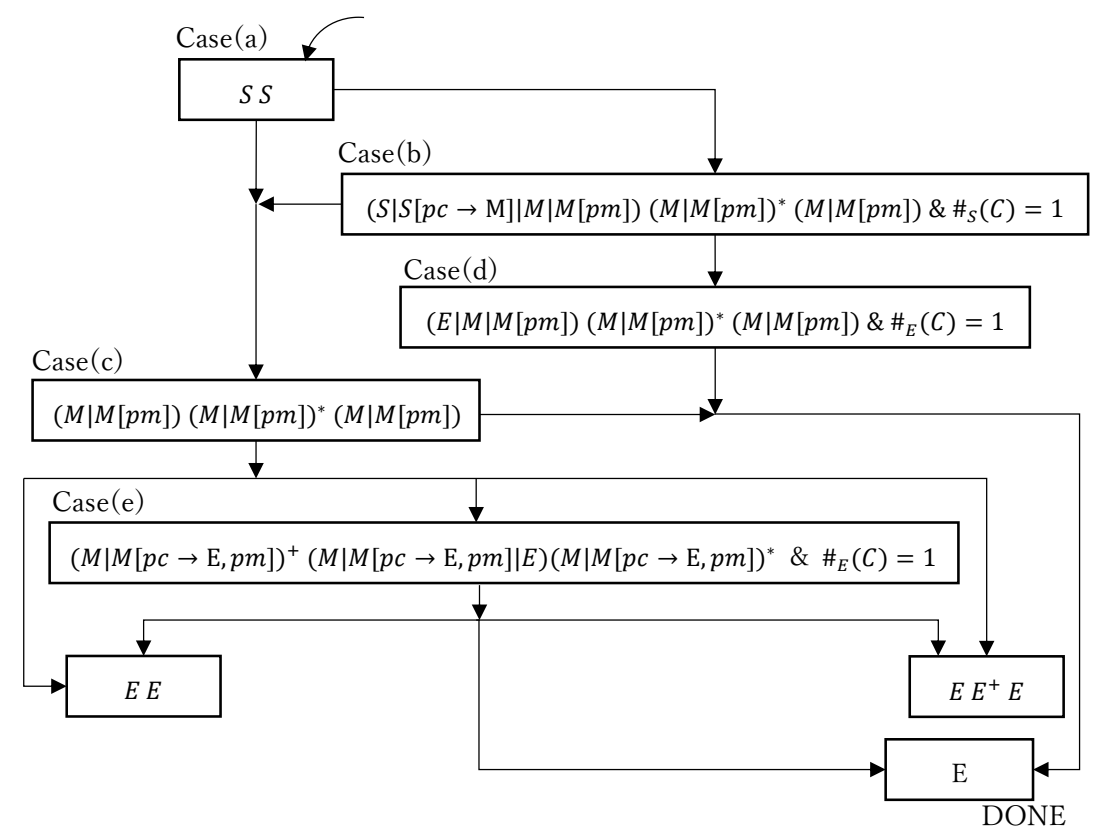

Fig. 6. Transition Graph for $\mathcal{L U M \mathcal { I }}$-Gather-in-ASYNC from $c c(\mathcal{C})=S S$.

$c c\left(\mathcal{C}\left(t_{S}\right)\right)=(S|S[p c \rightarrow M]| M \mid M[p m])(M \mid M[p m])^{*}(M \mid M[p m]), \#{ }_{S}\left(\mathcal{C}\left(t_{S}\right)\right)=1$ and the destination of robots in $M[p m]$ or $S[p c \rightarrow M]$ is the midpoint of onLDS in $\mathcal{C}(t)$ (Case (b)).

Let $p_{m}$ be the the midpoint of onLDS in $\mathcal{C}(t)$. As long as the both endpoints have $S$, robots with $S$ change their colors to $M$ and will move to $p_{m}$ as the destination (lines 5-6), and robots with $M$ do nothing when observing a configuration in $\forall S, M$. Thus, if there are no $S$ at the both endpoints at the same time, setting time $t_{\forall M}$ be the last $\operatorname{robot}(\mathrm{s})$ with $S$ perform Compute, $c c\left(\mathcal{C}\left(t_{\forall M}\right)\right)=(M \mid M[p m])(M \mid M[p m])^{*}(M \mid M[p m])$, because robots with $M$ do nothing when observing a configuration in $\forall S, M$ (line 18). Otherwise, there exists a time when one of the endpoints still has $S$ or $S[p c \rightarrow M]$ but the other endpoint does not have $S$. Setting time $t_{S}$ be such time, $c c\left(\mathcal{C}\left(t_{S}\right)\right)=(S \mid S[p c \rightarrow$ $M]|M| M[p m])(M \mid M[p m]) *(M \mid M[p m])$ and $\#_{S}\left(\mathcal{C}\left(t_{S}\right)\right)=1$.

Case (b) Transition from $c c\left(\mathcal{C}\left(t_{S}\right)\right)=(S|S[p c \rightarrow M]| M \mid M[p m])(M \mid M[p m])^{*}(M \mid M[p m])\left(t_{S}>t\right)$ and $\#_{S}\left(\mathcal{C}\left(t_{S}\right)\right)=1$

We show that in this case there is a time $t_{\forall M}\left(>t_{S}\right)$ such that $c c\left(\mathcal{C}\left(t_{\forall M}\right)\right)=$ $(M \mid M[p m])(M \mid M[p m])^{*}(M \mid M[p m])\left(\right.$ Case (c)) or a time $t_{G P}\left(>t_{S}\right)$ such that $c c(\mathcal{C}(G P))=(E|M| M[p m])(M \mid M[p m])^{*}(M \mid M[p m])$ and $\#_{E}\left(\mathcal{C}\left(t_{G P}\right)\right)=1$

(Case (d)). 
In the case that $c c\left(\mathcal{C}\left(t_{S}\right)\right)=(S[p c \rightarrow M]|M| M[p m])(M \mid M[p m])^{*}(M \mid M[p m])$, robots in $S[p c \rightarrow M]$ change their colors to $M$ and move to $p_{m}$. Furthermore, if robots with $M$ finish moving and become active again, they do nothing as long as $S$ exists. Therefore, there is a time $t_{\forall M}$ when $c c\left(\mathcal{C}\left(t_{\forall M}\right)\right)=$ $(M \mid M[p m])(M \mid M[p m])^{*}(M \mid M[p m])$, where robots in $M[p m]$ will move to $p_{m}$. Otherwise, there is a robot with $S$ that observes only one point with $S$, and the robot change its color to $E$ even if the color configuration is $(S|M| E) M^{*} M$. Furthermore, if robots with $M$ finish move and become active again, they do nothing as long as $S$ exists. Therefore, there is a time $t_{G P}$ when $c c\left(\mathcal{C}\left(t_{G P}\right)\right)=$ $(E|M| M[p m])(M \mid M[p m])^{*}(M \mid M[p m])$ and $\#_{E}\left(\mathcal{C}\left(t_{G P}\right)\right)=1$.

Case (c) Transition from $c c\left(\mathcal{C}\left(t_{\forall M}\right)\right)=(M \mid M[p m])(M \mid M[p m])^{*}(M \mid M[p m])$ $\left.\left(t_{\forall M}>t_{S}>t\right)\right)$

We show that if $c c\left(\mathcal{C}\left(t_{\forall M}\right)\right)=(M \mid M[p m])(M \mid M[p m])^{*}(M \mid M[p m])$, there is a time $t^{\prime}\left(>t_{\forall M}\right)$ such that $c c\left(\mathcal{C}\left(t^{\prime}\right)\right)=E$, a time $t_{E}\left(>t_{\forall M}\right)$ such that $c c\left(\mathcal{C}\left(t_{E}\right)\right)=$ $(M \mid M[p m, p c \rightarrow E])^{+}(E|M| M[p m, p c \rightarrow E])(M \mid M[p m, p c \rightarrow E])^{*}$ and $\#_{E}\left(\mathcal{C}\left(t_{E}\right)\right)=1($ Case $(\mathbf{e}))$, or a time $t^{\prime}\left(>t_{\forall M}\right)$ such that $c c\left(\mathcal{C}\left(t^{\prime}\right)=E E^{*} E\right.$.

If all robots moved to $p_{m}$ or are moving toward the same destination $p_{m}$ since all robots reach $p_{m}$, the color-configuration becomes $M$ or $(M \mid E)$. Then, robots with $M$ change their color to $E$ until the color-configuration becomes $E$ by Lemma 22 . Thus, there is a time $t^{\prime}$ such that $c c\left(\mathcal{C}\left(t^{\prime}\right)\right)=E$.

If there are robots at only one point that observe $c c(\mathcal{S S})=M M^{*} M$ and perform Compute, the robots change their colors to $E$. Robots on points except the point may observe $c c(\mathcal{S S})=M M^{*} M$ but do not change their colors. Setting time $t_{E}$ be such time, $c c\left(\mathcal{C}\left(t_{E}\right)=(M \mid M[p m, p c \rightarrow E])^{+}(E|M| M[p m, p c \rightarrow\right.$ $E])(M \mid M[p m, p c \rightarrow E])^{*}$. Otherwise, there are robots at one or more different points that observe $c c(\mathcal{S S})=M M^{*} M$ and change their colors to $E$. Then, the configuration satisfies $\#_{E}(\mathcal{C}) \geq 2$. Since all robots with $M$ become active by the fairness of ASYNC and change their colors to $E$ until the configuration in $\forall E$, there is a time $t^{\prime}$ when the color-configuration becomes $E E^{*} E$. In this case, all robots have toward the midpoint $p_{m}$ of $\operatorname{LDS}$ in $\mathcal{C}(t)$, and changed their colors to $E$. Therefore, $\operatorname{dis}\left(\mathcal{C}\left(t^{\prime}\right)\right)$ is at least $2 \delta$ shorter than $\operatorname{dis}(\mathcal{C}(t))$.

Case (d) Transition from $c c\left(\mathcal{C}\left(t_{G P}\right)\right)=(E|M| M[p m])(M \mid M[p m])^{*}(M \mid M[p m])$ $\left(t_{G P}>t_{S}>t\right)$ and $\#_{E}\left(\mathcal{C}\left(t_{G P}\right)\right)=1$.

If $c c\left(\mathcal{C}\left(t_{G P}\right)\right)=(E|M| M[p m])(M \mid M[p m])^{*}(M \mid M[p m])$ and $\#_{E}\left(\mathcal{C}\left(t_{G P}\right)\right)=$ 1 , there is a time $t^{\prime}\left(>t_{G P}\right)$ such that $c c\left(\mathcal{C}\left(t^{\prime}\right)\right)=E$. Let $p_{G P}$ be a position of the robot with $E$ at $t_{G P}$. Robots not on $p_{G P}$ have colors $M$ and be moving toward $p_{m}$ or have finished moving. They move toward $p_{G P}$ when observing this configuration. It is possible that robots with $M[p m]$ on $p_{G P}$ move toward $p_{m}$ and leave $p_{G P}$. However, when they are not on $p_{G P}$, they perform the same action as previously described. Robots with $E$ on $p_{G P}$ do nothing. Thus, the number of positions where a robot has color $\mathrm{E}$ do not change, the number of robots with $M[p m]$ decreases. Also, since the number of robots with $\mathrm{E}$ increases when $(M \mid E)$, following from Lemma 22, there is a time $t^{\prime}$ such that $c c\left(\mathcal{C}\left(t^{\prime}\right)\right)=E$. 
Case (e) Transition from $c c\left(\mathcal{C}\left(t_{E}\right)\right)=(M \mid M[p m, p c \rightarrow E])^{+}$ $(E|M| M[p m, p c \rightarrow E])(M \mid M[p m, p c \rightarrow E])^{*}\left(t_{E}>t_{\forall M}>t\right)$ and $\#_{E}\left(\mathcal{C}\left(t_{E}\right)\right)=1$.

We show that for these configurations there is a time $t^{\prime}\left(>t_{E}\right)$ such that $c c\left(\mathcal{C}\left(t^{\prime}\right)\right)=E E^{*} E$, or a time $t^{\prime}\left(>t_{E}\right)$ such that $c c\left(\mathcal{C}\left(t^{\prime}\right)\right)=E$.

Let $p_{E}$ be a position of the robot with $\mathrm{E}$ at $t_{E}$. If a robot not on $p_{E}$ observes between $t_{\forall M}$ and $t_{E}$, it changes its color to $E$. When it changes its color, the configuration becomes $\#_{E}(\mathcal{C}) \geq 2$. Since all robots with $M$ become active by the fairness of ASYNC and change their colors to $E$ until the configuration in $\forall E$, there is a time $t^{\prime}$ at which the color-configuration becomes $E E^{*} E$. In this case, all robots have toward the midpoint $p_{m}$ of LDS in $\mathcal{C}(t)$, and changed their colors to $E$. Therefore, $\operatorname{dis}\left(\mathcal{C}\left(t^{\prime}\right)\right)$ is at least $2 \delta$ shorter than $\operatorname{dis}(\mathcal{C}(t))$. Otherwise, robots that observes between $t_{\forall M}$ and $t_{E}$ are at only $p_{E}$. Because of the same argument of Case (d), there is a time $t^{\prime}$ such that $c c\left(\mathcal{C}\left(t^{\prime}\right)\right)=E$.

Lemma 17. If $c c(\mathcal{C}(t))=E E$, there is a time $t^{\prime}(>t)$ such that $c c\left(\mathcal{C}\left(t^{\prime}\right)\right)=S S$.

Proof. Robots change their colors to $S$ until the color-configuration becomes $S S$. Since all robots become active after $t$ by the fairness of ASYNC, there is a time $t^{\prime}$ at which the color-configuration becomes $S S$.

Lemma 18. If $c c(\mathcal{C}(t))=E E^{+} E$, there is a time $t^{\prime}(>t)$ such that $c c\left(\mathcal{C}\left(t^{\prime}\right)\right)=$ $E E_{m} E$.

Proof. If the color-configuration is $E E^{+} E$, robots at endpoints do nothing, and robots at points except the endpoints move to the midpoint $p_{m}$ of two endpoints in $\mathcal{C}(t)$. Therefore, moving robot's destination is fixed, and the colorconfiguration becomes $E E_{m} E$.

Lemma 19. If $c c(\mathcal{C}(t))=E E_{m} E$, there is a time $t^{\prime}(>t)$ such that $c c\left(\mathcal{C}\left(t^{\prime}\right)\right)=$ $S E_{m} S$.

Proof. If the color-configuration is $E E_{m} E$, robots at the endpoints change their colors to $S$, and robots at the midpoint do nothing. The color of robots with $E$ at the midpoint do not change until robots at the endpoints change their colors to $S$. Thus, the color-configuration becomes $S E_{m} S$.

Lemma 20. If $c c(\mathcal{C}(t))=S E_{m} S$, there is a time $t^{\prime}(>t)$ such that $c c\left(\mathcal{C}\left(t^{\prime}\right)\right)=$ $M E_{m} M$.

Proof. If the color-configuration is $S E_{m} S$, robots at the endpoints change their colors to $M$, and robots at the midpoint do nothing. The color of robots with $E$ at midpoint do not change until robots at the endpoints change their colors to $M$. Thus, the color-configuration becomes $M E_{m} M$.

Lemma 21. If $c c(\mathcal{C}(t))=M E_{m} M$, there is a time $t^{\prime}(>t)$ such that $c c\left(\mathcal{C}\left(t^{\prime}\right)\right)=$ E. 
Proof. Let $p_{E}$ be a position of the robot with $E$ at $t$. Robots not located on $p_{E}$ move to $p_{E}$. Since robots on $p_{E}$ do nothing, there is a time $t^{\prime}$ at which the color-configuration is $c c\left(\mathcal{C}\left(t^{\prime}\right)\right)=(M \mid E)$.

Lemma 22. If $c c(\mathcal{C}(t))=(M|M[p c \rightarrow E]| E)$ and $\#_{M}(\mathcal{C}(t))=1$, there is a time $t^{\prime}(>t)$ such that $c c\left(\mathcal{C}\left(t^{\prime}\right)\right)=E$.

Proof. Robots with $M$ change their colors to $E$ until the color-configuration becomes $E$. Meanwhile, robots with $E$ do nothing. Since all robots become active after $t$ by the fairness of ASYNC, there is a time $t^{\prime}$ at which the the colorconfiguration becomes $E$.

Lemma 23. If $c c(\mathcal{C}(t))=(S|S[p c \rightarrow M o r E]| M)$, \# ${ }_{S}(\mathcal{C}(t))=1$ and $\#_{M}(\mathcal{C}(t))=$ 1 , there is a time $t^{\prime}(>t)$ such that $c c\left(\mathcal{C}\left(t^{\prime}\right)\right)=(M \mid E)$ and $\#_{M}\left(\mathcal{C}\left(t^{\prime}\right)\right)=1$.

Proof. It is possible that robot with $S$ observes $c c(\mathcal{C}(t))$ or $c c(\mathcal{C})=(S|M| E)$, $\#_{M}(\mathcal{C}(t))=1$, and $\#_{E}(\mathcal{C}(t))=1$ by the behavior of $S[p c]$. If a robot with $S$ observes $\mathcal{C}(t)$, the robot changes its color to $E$. If a robot with $S$ observes $c c(\mathcal{C})=(S|M| E), \#_{M}(\mathcal{C}(t))=1$, and $\#_{E}(\mathcal{C}(t))=1$, the robot changes its color to $E$. In both case, robots with $M$ or $E$ do nothing until $\#_{S}(\mathcal{C})$ is 0 . Therefore, there is a time $t^{\prime}$ such that $c c\left(\mathcal{C}\left(t^{\prime}\right)\right)=(M \mid E)$ and $\#_{M}\left(\mathcal{C}\left(t^{\prime}\right)\right)=1$.

We show that Algorithm 5 can work from the configurations in Lemma 14.

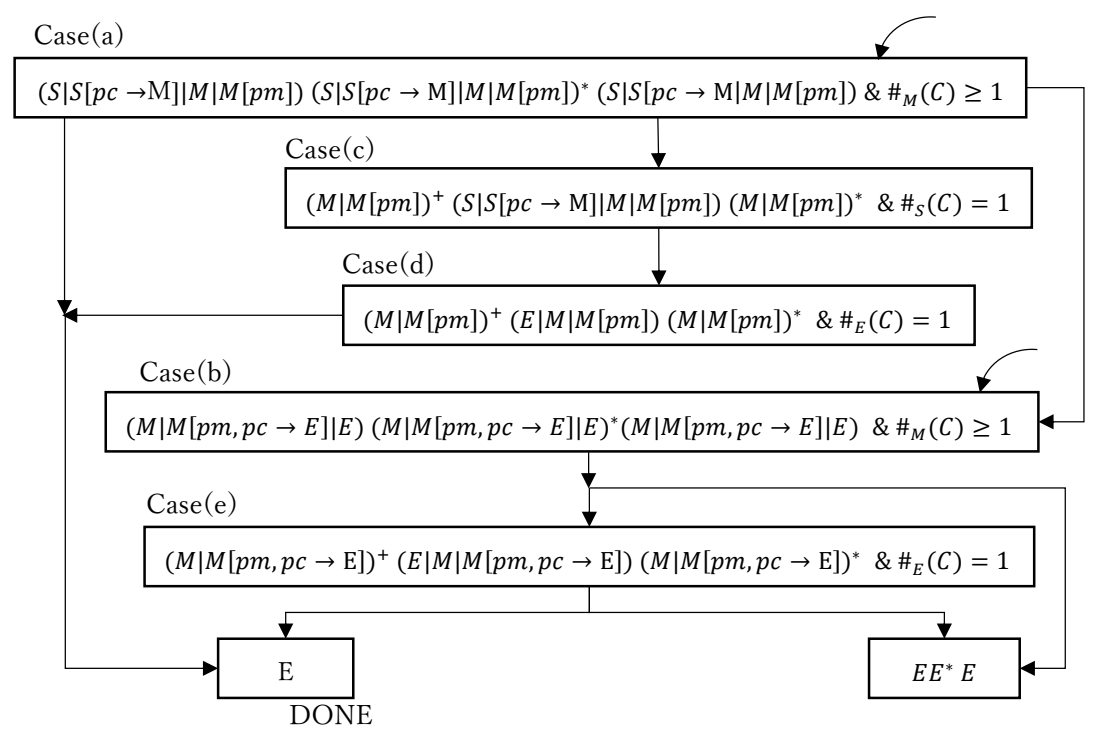

Fig. 7. Transition Graph after switching from simulation of ElectOneLDS (Lemma 24) 
Lemma 24. If $c c(\mathcal{C}(t))$ is one of (1)-(5) in Lemma 14, then there is a time $t^{\prime}(\geq t)$ such that the color-configuration satisfies (a) or (b) in the followings,

(a) $c c\left(\mathcal{C}\left(t^{\prime}\right)\right)=E E^{*} E$,

(b) $c c\left(\mathcal{C}\left(t^{\prime}\right)\right)=E$.

Proof. For (1), we can prove it by Lemmas 15 and 16. For (4) and (5), we can prove them by Lemmas 23 and 22, respectively.

For (2) and (3), we can prove it in the followings. Transitions from configurations (2) and (3) are depicted in Fig. 7.

- $\operatorname{Case}(\mathbf{a}) c c(\mathcal{C}(t))=$

$(S|S[p c \rightarrow M]| M \mid M[p m])(S|S[p c \rightarrow M]| M \mid M[p m]) *(S|S[p c \rightarrow M]| M \mid M[p m])$ and $\#_{M}(\mathcal{C}(t)) \geq 1$

We show that if Case (a) holds, there is a time $t_{\forall M}(\geq t)$ such that $c c\left(\mathcal{C}\left(t_{\forall M}\right)\right)=$ $(M|M[p m, p c \rightarrow E]| E)(M|M[p m, p c \rightarrow E]| E)^{*}(M|M[p m, p c \rightarrow E]| E)$ (Case (b)), a time $t_{S}(\geq t)$ such that $c c\left(\mathcal{C}\left(t_{S}\right)\right)=(M \mid M[p m])^{+}$

$(S|S[p c \rightarrow M]| M \mid M[p m])(M \mid M[p m])^{*}$ and $\#_{S}(\mathcal{C}(t))=1($ Case $(\mathbf{c}))$, or there is a time $t^{\prime}(>t)$ such that $c c\left(\mathcal{C}\left(t^{\prime}\right)\right)=E$. Note that $c c(\mathcal{C}(t))$ may be in Case (b) or Case (c).

In the case of $\#_{S}(\mathcal{C}(t)) \geq 3$, robots with $S$ change their colors to $M$. Thus, $\#_{S}(\mathcal{C}(t))$ is decreasing until $\#_{S}(\mathcal{C}) \leq 2$.

If $\#_{S}(\mathcal{C}(t))=2$, and the both endpoints of onLDS have $S$, robots with $S$ change their colors to $M$ and move to the midpoint of onLDS. Thus, $\#_{S}(\mathcal{C}(t))$ decreases to 0 or 1.

If $\#_{S}(\mathcal{C}(t))=2$, and robots with $S$ is not on the both endpoints of on, robots with $S$ change their color to $M$. Thus, $\#_{S}(\mathcal{C}(t))$ decreases to 0 or 1 .

If $\#_{S}(\mathcal{C}(t))=1$, there is a time $t_{S}(>t)$ when $c c\left(\mathcal{C}\left(t_{S}\right)\right)=(M \mid M[p m])^{+}$ $(S|S[p c \rightarrow M]| M[p m])(M \mid M[p m])^{*}$, or there is a time $t_{o p}(>t)$

when $c c\left(\mathcal{C}\left(t_{o p}\right)\right)=(S|S[p c \rightarrow M]| M)$. In the case of $c c\left(\mathcal{C}\left(t_{o p}\right)\right)=(S \mid S[p c \rightarrow$ $M] \mid M), \mathcal{C}\left(t^{\prime \prime}\right)\left(t^{\prime \prime}=\max \left\{t_{s c} \mid \mathcal{C}\left(t_{s c}\right) \in \forall S, t_{s c}<t\right\}\right)$ is symmetric and contractible. Robots with $M[p m]$ that executed ElectOneLDS at $t^{\prime \prime}$ have same destination that is the center of $\mathcal{C H}\left(\mathcal{C}\left(t^{\prime \prime}\right)\right)$. Therefore, there is a case of $(S|S[p c \rightarrow M]| M)$. We can obtain from Lemma 22 and Lemma 23 that there is a time $t^{\prime}$ such that $c c\left(\mathcal{C}\left(t^{\prime}\right)\right)=E$.

If $\#_{S}(\mathcal{C}(t))=0$, robots with $M$ change their colors to $E$. Thus, there is a time $t_{\forall M}$ when $(M|M[p m, p c \rightarrow E]| E)(M|M[p m, p c \rightarrow E]| E)^{*}(M \mid M[p m, p c \rightarrow$ $E] \mid E)$.

- Case (b) $c c(\mathcal{C}(t))=$ $(M|M[p m, p c \rightarrow E]| E)(M|M[p m, p c \rightarrow E]| E)^{*}(M|M[p m, p c \rightarrow E]| E)$ and $\#_{M}(\mathcal{C}(t)) \geq 1$

We show that if Case (b) holds, there is a time $t_{E}(\geq t)$ such that $c c\left(\mathcal{C}\left(t_{E}\right)\right)=$ $(M \mid M[p c \rightarrow E, p m])^{+}(M|M[p m, p c \rightarrow E]| E)(M \mid M[p m, p c \rightarrow E])$ and $\left.\#_{E}\left(\mathcal{C}\left(t_{E}\right)\right)=1\right)($ Case $(\mathbf{e}))$ or a time $t^{\prime}(>t)$ such that $c c\left(\mathcal{C}\left(t^{\prime}\right)\right)=E E^{*} E$. Note that $c c(\mathcal{C}(t))$ may be in Case $(\mathbf{e})$ or $c c(\mathcal{C}(t))=E E^{*} E$. 
In the case of $\#_{E}(\mathcal{C}(t))=0$, robots with $M$ change their colors to $E$. If there are robots at only one point that recognize $\#_{E}(\mathcal{C}(t))=0$, there is a time $t_{E}$ when $(M \mid M[p m, p c \rightarrow E])^{+}(M|M[p m, p c]| E)(M \mid M[p m, p c \rightarrow E])^{*}$ and $\#_{E}\left(\mathcal{C}\left(t_{E}\right)=1\right.$.

If there are robots at one or more points in different position that recognize $\#_{E}(\mathcal{C}(t))=0, \#_{E}(\mathcal{C}(t))$ is increasing to 2 or more. Then, robots with $M$ change their colors to $E$ until the configuration in $\forall E$. The same behavior occurs when $\#_{E}(\mathcal{C}(t)) \geq 2$. Therefore, there is a time $t^{\prime}$ when $E E^{*} E$.

- Case (c) $c c\left(\mathcal{C}\left(t_{S}\right)\right)=(M \mid M[p m])^{+}(S|S[p c \rightarrow M]| M[p m])(M \mid M[p m])^{*}$ and $\#_{S}(\mathcal{C}(t))=1\left(t_{S} \geq t\right)$

We show that if Case (c) occurs, there is a time $t_{\forall M}\left(>t_{S}\right)$ such that $c c\left(\mathcal{C}\left(t_{\forall M}\right)\right)=(M|M[p m, p c \rightarrow E]| E)(M|M[p m, p c \rightarrow E]| E)^{*}(M \mid M[p m, p c \rightarrow$ $E] \mid E)\left(\right.$ Case (b)) or a time $t_{G P}\left(>t_{S}\right)$ such that $c c\left(\mathcal{C}\left(t_{G P}\right)\right)=(M \mid M[p m])^{+}$ $(E|M| M[p m])(M \mid M[p m])^{*}$ and $\#_{E}\left(\mathcal{C}\left(t_{G P}\right)\right)=1($ Case $(\mathbf{d}))$.

If all robots with $S$ perform Look before $t_{S}$, they change their colors to $M$ and may move to a point on onLDS. Furthermore, if robots with $M$ finish moving and become active again, they do nothing as long as $S$ exists. Therefore, $\mathcal{C}\left(t_{S}\right)$ becomes a configuration in $\forall M$. Then, since robots with $M$ change their colors to $E$, there is a time $t_{\forall M}$ when $(M \mid M[p m, p c \rightarrow$ $E] \mid E)(M|M[p m, p c \rightarrow E]| E)^{*}(M|M[p m, p c \rightarrow E]| E)$.

there is a time $t_{\forall M}$ when $c c\left(\mathcal{C}\left(t_{\forall M}\right)\right)=(M \mid M[p m])(M \mid M[p m])^{*}$

$(M \mid M[p m])$. Otherwise, there is a robot with $S$ that does not perform Look before $t_{S}$, and it changes its color to $E$ when observing $\#_{S}(\mathcal{C})=1$. Furthermore, if robots with $M$ finish moving and become active again, they do nothing as long as $S$ exists. Therefore, there is a time $t_{E}$ when $c c\left(\mathcal{C}\left(t_{G P}\right)\right)=$ $(M \mid M[p m])^{+}(E|M| M[p m])(M \mid M[p m])^{*}$ and $\#_{E}\left(\mathcal{C}\left(t_{G P}\right)\right)=1$.

- Case (d) $c c\left(\mathcal{C}\left(t_{G P}\right)\right)=(M \mid M[p m])^{+}(E|M| M[p m])(M \mid M[p m])^{*}$ and $\#_{E}\left(\mathcal{C}\left(t_{G P}\right)\right)=1\left(t_{G P}>t_{S}\right)$

We show that if Case (d) occurs, there is a time $t^{\prime}\left(>t_{G P}\right)$ such that $\mathcal{C}\left(t^{\prime}\right)$ is Gathering configuration. Let $p_{G P}$ be a position having robot with $E$ at time $t_{G P}$. Robots not on $p_{G P}$ have color $M$ and may move toward a point on onLDS or be done moving. They move toward $p_{G P}$ when observing this configuration. It is possible that robots with $M[p m]$ on $p_{G} P$ move toward $p_{m}$ and leave $p_{G P}$. However, when they are not on $p_{G P}$, they perform the same action as previously described. Robots with $E$ on $p_{G P}$ do nothing. Thus, the number of positions having robots have color $E$ do not change, and so there is a time $t^{\prime}$ such that $c c\left(\mathcal{C}\left(t^{\prime}\right)\right)=E$ by lemma 22 .

- Case (e) $c c\left(\mathcal{C}\left(t_{E}\right)\right)=(M \mid M[p c \rightarrow E, p m])^{+}$ $(M|M[p m, p c \rightarrow E]| E)(M \mid M[p m, p c \rightarrow E])$ and $\#_{E}\left(\mathcal{C}\left(t_{E}\right)\right)=1\left(t_{E}>t_{\forall M}\right)$ We show that if Case (e) occurs, there is a time $t^{\prime}\left(>t_{E}\right)$ such that $c c\left(\mathcal{C}\left(t^{\prime}\right)\right)=$ $E E^{*} E$, a time $t^{\prime}\left(>t_{E}\right)$ such that $\mathcal{C}\left(t^{\prime}\right)$ is a Gathering configuration.

Let $p_{E}$ be the position having robots with $E$ at time $t_{E}$. If there is a robot not on $p_{E}$ that observed between $t_{\forall M}$ and $t_{E}$, it changes its color to $E$. When it changes the color, the configuration has $\#_{E}(\mathcal{C}) \geq 2$. Since all robots with $M$ become active by ASYNC scheduler's fairness and change their colors to $E$ until the configuration in $\forall E$, there is a time $t^{\prime}$ when the color- 
configuration becomes $E E^{*} E$. Otherwise, robots that observed between $t_{\forall M}$ and $t_{E}$ are located at only $p_{E}$. Because of the same argument of transition from $(E|M| M[p m])(M \mid M[p m])^{*}(M \mid M[p m])$ and $\#_{E}(\mathcal{C})=1$, there is a time $t^{\prime}$ when $\operatorname{cc}\left(\mathcal{C}\left(t^{\prime}\right)\right)=E$.

Lemmas 14-24 follow the following theorem and we obtain our main result.

Theorem 5. $\mathcal{L U} \mathcal{M I}$-Gather-in-ASYNC solves Gathering from onLDS for $\mathcal{L U M \mathcal { I }}$ robots having 3 colors, under non-rigid movement.

Theorem 6. Gathering can be solved in $A S Y N C$ by $\mathcal{L U M \mathcal { I }}$ robots having 3 colors under non-rigid movement and agreement of chirality.

\section{Concluding Remarks}

We have shown a Gathering algorithm in non-rigid and ASYNC with $\mathcal{L U M \mathcal { I }}$ of three colors. In order to obtain the algorithm, we have shown a simulating algorithm of any algorithm in unfair SSYNC by $\mathcal{L U M \mathcal { I }}$ of three colors in ASYNC. We have reduced the number of colors used in the simulation to three from five, although the simulated algorithms are ones in unfair SSYNC.

The method by combining the simulation of SSYNC robots by ASYNC ones and algorithms working in SSYNC not only reduces the number of colors used in the resultant algorithm but also simplifies the proof of correctness of it. As is known from an example of ElectOneLDS, it seems to be very complicated to extend ElectOneLDS such that it can work in ASYNC and prove the correctness. However, about correctness of the combined algorithm, it is enough to prove the correctness of the simulation working in ASYNC because the correctness of ElectOneLDS working in SSYNC has been obtained.

One of the interesting open questions is the number of colors to solve Gathering in ASYNC, although two colors are enough to solve Rendezvous in ASYNC[18], we conjecture that three colors are necessary to solve Gathering in ASYNC.

Acknowledgment This research was partly supported by JSPS KAKENHI No. 20H04140, 20KK0232, 20K11685, 21K11748, and by Japan Science and Technology Agency (JST) SICORP Grant\#JPMJSC1806.

\section{References}

1. N. Agmon and D. Peleg. Fault-tolerant gathering algorithms for autonomous mobile robots. SIAM Journal on Computing, 36(1):56-82, 2006.

2. H. Ando, Y. Osawa, I. Suzuki, and M. Yamashita. A distributed memoryless point concergence algorithm for mobile robots with limited visivility. IEEE Transactions on Robotics and Automation, 15(5):818-828, 1999.

3. S. Bhagat and K. Mukhopadhyaya. Optimum gathering of asynchronous robots. In Algorithms and Discrete Applied Mathematics (CALDAM 2017), pages 37-49, 2017. 
4. Z. Bouzid, S. Das, and S. Tixeuil. Gathering of mobile robots tolerating multiple crash faults. In the 33rd Int. Conf. on Distributed Computing Systems, pages 334$346,2013$.

5. S. Cicerone, G. Di Stefano, and A. Navarra. "semi-asynchronous": A new scheduler in distributed computing. IEEE Access, 9:41540-41557, 2021.

6. M. Cieliebak, P. Flocchini, G. Prencipe, and N. Santoro. Distributed computing by mobile robots: Gathering. SIAM Journal on Computing, 41(4):829-879, 2012.

7. S. Das, P. Flocchini, G. Prencipe, N. Santoro, and M. Yamashita. Autonomous mobile robots with lights. Theoretical Computer Science, 609:171-184, 2016.

8. X. Défago, A. Hériban, S. Tixeuil, and K. Wada. Using model checking to formally verify rendezvous algorithms for robots with lights in euclidean space. In 2020 International Symposium on Reliable Distributed Systems (SRDS), pages 113-122, 2020.

9. X. Défago, M. Gradinariu Potop-Butucaru, J. Clément, S. Messika, and P. Raipin Parvédy. Fault and byzantine tolerant self-stabilizing mobile robots gathering feasibility study. CoRR abs/1602.05546, arXiv, 2016.

10. B. Degener, B. Kempkes, T. Langner, F. Meyer auf der Heide, P. Pietrzyk, and R. Wattenhofer. A tight run-time bound for synchronous gathering of autonomous robots with limited visibility. In 23rd ACM SPAA, pages 139-148, 2011.

11. M. D'Emidio, D. Frigioni, and A. Navarro. Synchronous robots vs asynchronous lights-enhanced robots on graphs. Electr. Notes Theor. Comput. Sci., 322:169-180, 2016.

12. M. D'Emidio, G. Di Stefano, D. Frigioni, and A. Navarra. Characterizing the computational power of mobile robots on graphs and implications for the euclidean plane. Inf. Comput., 263:57-74, 2018.

13. G.A. Di Luna, P. Flocchini, S.G. Chaudhuri, F. Poloni, N. Santoro, and G. Viglietta. Mutual visibility by luminous robots without collisions. Information and Computation, 254(3):392-418, 2017.

14. Y. Dieudonné and F. Petit. Self-stabilizing gathering with strong multiplicity detection. Theoretical Computer Science, 428(13):47-57, 2012.

15. P. Flocchini, G. Prencipe, and N. Santoro. Distributed Computing by Oblivious Mobile Robots. Morgan \& Claypool, 2012.

16. P. Flocchini, G. Prencipe, and N. Santoro, editors. Distributed Computing by Mobile Entities, Current Research in Moving and Computing, volume 11340 of Lecture Notes in Computer Science. Springer, 2019.

17. P. Flocchini, N. Santoro, G. Viglietta, and M. Yamashita. Rendezvous with constant memory. Theoretical Computer Science, 621:57-72, 2016.

18. A. Hériban, X. Défago, and S. Tixeuil. Optimally gathering two robots. In Proc. of 19th Int. Conference on Distributed Computing and Networking (ICDCN), pages $1-10,2018$.

19. T. Izumi, Y. Katayama, N. Inuzuka, and K. Wada. Gathering autonomous mobile robots with dynamic compasses: An optimal result. In 21st DISC, pages 298-312, 2007.

20. T. Izumi, S. Souissi, Y. Katayama, N. Inuzuka, X. Défago, K. Wada, and M. Yamashita. The gathering problem for two oblivious robots with unreliable compasses. SIAM Journal on Computing, 41(1):26-46, 2012.

21. S. Kamei, A. Lamani, F. Ooshita, and S. Tixeuil. Asynchronous mobile robot gathering from symmetric configurations without global multiplicity detection. In 18th SIROCCO, pages 150-161, 2011.

22. R. Klasing, E. Markou, and A. Pelc. Gathering asynchronous oblivious mobile robots in a ring. Theoretical Computer Science, 390(1):27-39, 2008. 
23. J. Lin, A.S. Morse, and B.D.O. Anderson. The multi-agent rendezvous problem. parts 1 and 2. SIAM Journal on Computing, 46(6):2096-2147, 2007.

24. G. Prencipe. Impossibility of gathering by a set of autonomous mobile robots. Theoretical Computer Science, 384(2-3):222-231, 2007.

25. S. Souissi, X. Défago, and M. Yamashita. Using eventually consistent compasses to gather memory-less mobile robots with limited visibility. ACM Transactions on Autonomous and Adaptive Systems, 4(1):1-27, 2009.

26. I. Suzuki and M. Yamashita. Distributed anonymous mobile robots: Formation of geometric patterns. SIAM Journal on Computing, 28:1347-1363, 1999.

27. S. Terai, K. Wada, and Y. Katayama. Gathering problems for autonomous mobile robots with lights. arXiv.org, cs(ArXiv:1811.12068), 2018.

28. G. Viglietta. Rendezvous of two robots with visible bits. In 10th International Symposium on Algorithms and Experiments for Sensor Systems, Wireless Networks and Distributed Robotics (ALGOSENSORS), pages 291-306, 2013. 